\title{
Reconstruction of Two-Dimensional Signals from Level Crossings
}

\author{
AVIDEH ZAKHOR, MEMBER, IEEE, AND ALAN V. OPPENHEIM, FELLOW, IEEE
}

Invited Paper

Recent results indicate the reconstruction of two-dimensional signals from crossings of one level requires, in theory and practice, extreme accuracy in positions of the samples. The representation of signals with one-level crossings can be viewed as a trade-off between bandwidth and dynamic range, in the sense that if the available bandwidth is sufficient to preserve the level crossings accurately, then the dynamic range requirements are significantly reduced. On the other hand, representation of signals via their samples at the Nyquist rate can be considered as requiring relatively small bandwidth and large dynamic range. This is because, at least in theory, amplitude information at prespecified points are needed, to infinite precision. Sampling and reconstruction schemes are derived whose characteristics lie between these two extremes. First, an overview of existing results in zero crossing representation is presented, and next a number of new results on sampling schemes for reconstruction from multiple-level threshold crossing are developed. The quantization characteristics of these sampling schemes appear to lie between those of Nyquist sampling and one-level crossing representations, thus bridging the gap between explicit Nyquist sampling, and implicit one-level crossing sampling strategies.

\section{INTRODUCTION}

Signal reconstruction from partial information plus constraints has been an active area of research for many years. Reconstruction of bandlimited signals from Nyquist samples is, of course, a classical and extremely important example. Another, which has been the basis for considerable research, is reconstruction of a signal from its zero crossings ${ }^{1}$ and, more generally, from its crossings of a prespecified function such as a constant threshold or a sinusoid [1]-[7]. Other examples include reconstruction of onedimensional or multidimensional signals from Fourier transform magnitude, phase, or signed-magnitude information [8]-[12]. The variety of results on signal reconstruction have not only had a major impact in fields such as signal processing, communication theory and information theory, but also have been extensively applied to problems in

Manuscript received September 6, 1988; revised July 28, 1989. A. Zakhor is with the Department of Electrical Engineering and Computer Sciences, University of California, Berkeley, CA 94720.

A. V. Oppenheim is with the Department of Electrical Engineering and Computer Science, Massachusetts Institute of Technology, Cambridge, MA 02139.

IEEE Log Number 8933330.

${ }^{1}$ By zero crossings of a signal we mean the values of the independent variable at which the signal becomes zero. such diverse areas as optics, radio astronomy, crystallography, tomography, geophysics and oceanography.

Nyquist sampling and signal representation in terms of zero crossings both correspond to sampling strategies. Sampling methods based on prespecified sampling instants, such as Nyquist sampling, are often referred to as explicit [7]. Sampling strategies such as zero crossings, or crossings of a prespecified function, in which a representation is sought in terms of the instants in which the signal assumes prespecified values, are referred to as implicit. The great majority of research in this area has been in communication theory, and has concentrated on one-dimensional signals. For example, Bond and Cahn [2] considered representation and manipulation of one-dimensional signals by means of their real and complex zeros. Extensive work on this topic has been done by Voelcker [4], who shows how modulation processes can be considered to be methods of manipulating or extracting the zeros of a signal and that different systems can be analyzed as to how they affect the zeros of a signal. Computer simulation of these results has been reported by Sekey [3]. Logan developed a new class of bandpass signals which are uniquely specified by their real zero crossings [5]. Specifically, he showed that almost all bandpass signals of bandwidth less than one octave are uniquely specified by their real zero crossings. In addition, Bar-David [7] considered the important case of one-dimensional implicit sampling in terms of real variables alone. These results have been used to overcome distortions that are incurred either by intentional nonlinear processing or by inadvertent nonlinearities. Such nonlinearities might arise in single sideband systems, where the modulating signal is typically hardlimited to decrease its dynamic range. Also, in magnetic tape recording, a strong higher frequency bias tone is usually added onto the signal to ensure fidelity in the presence of inherent material nonlinearity.

From a practical point of view, implicit sampling also has potential applications in multidimensional problems in image processing and vision. For instance, it has been suggested that image representation in vision may be accomplished by extraction of zero crossings [6]. Also, a number of papers in vision stress the importance of the information contained in the edges of objects which, from a computational point of view, correspond to the zero crossings of the Laplacian of the Gaussian of the image [14]. Further- 
more, one theory of human vision relies primarily on edge detection as the mechanism by which humans process visual information. There are also a number of applications in which it is desired to recover a signal from its threshold crossings. For instance, an image which has been corrupted by a memoryless nonlinear distortion containing at least one monotonic region, can be recovered from threshold crossings of its distorted version [15]. This could be potentially useful in applications in which intensity levels of images recorded on film are likely to become distorted over time but for which threshold crossing information could be preserved.

From a theoretical point of view, there are two major results in implicit sampling of multidimensional signals, both of which deal with reconstruction from zero crossings. Specifically, Rotem and Zeevi [16] extended Logan's one-dimensional result for bandpass signals to two dimensions, and Curtis and Oppenheim [1] found conditions under which bandlimited periodic (BLP) signals can be reconstructed from their crossings of a single prespecified threshold (e.g., a threshold of zero for the specific case of zero crossings, or one-level crossings more generally). Although the latter results are more general than those based on the extension of one-dimensional results, the accuracy with which the locations of the one-level crossings need to be specified is large enough to limit applicability of the results in many practical situations. Specifically, in theory, the positions of the crossings must be known to infinite precision, and in practice, with extreme accuracy.

The representation of signals with one-level crossings can be viewed as a trade-off between bandwidth and dynamic range, in the sense that if the available bandwidth is sufficient to preserve the level crossings accurately, then the dynamic range requirements are significantly reduced. On the other hand, representation of signals via their samples at the Nyquist rate can be considered as requiring relatively small bandwidth and large dynamic range. This is because, in theory, exact recovery of signals via Nyquist sampling requires amplitude information at prespecified points, to infinite precision. Thus the natural question which arises is whether or not there are intermediate sampling and reconstruction schemes whose characteristics lie between these two extremes. In this paper, we review results on twodimensional signal reconstruction from zero crossings and develop a number of new results on sampling schemes for reconstruction from multiple-level threshold crossings. As we will see, the quantization characteristics of these sampling schemes appear to lie between those of Nyquist sampling and one-level crossing representations, thus bridging the gap between explicit and implicit sampling strategies.

The outline of the paper is as follows. In section II we review the two-dimensional zero crossings results of Rotem and Zeevi [16] and of Curtis and Oppenheim [1]. In section III, we derive semi-implicit and implicit sampling strategies for reconstruction of signals from multiple-level threshold crossings. As stated previously, in implicit sampling methods a signal is represented in terms of the points at which it assumes prespecified values on prespecified curves. An example of semi-implicit sampling would be the set of samples representing the intersections of all zero crossing contours with a circle. As we will see in section III, the semiimplicit sampling results can be used not only to reconstruct signals from their multiple level crossings, but also to recover them from their crossings with arbitrary functions, or from nonuniformly spaced samples.

A problem distinct from that of uniquely specifying signals with level crossings is that of developing specific algorithms for recovering them from level crossing information, once it is known that the signals satisfy the appropriate constraints. In section IV, we propose a variety of reconstruction algorithms for each of the two sampling strategies of section III, and demonstrate the results for several images. Section $V$ includes a preliminary investigation of the quantization characteristics of some of the proposed sampling and reconstruction schemes. Finally, conclusions and future directions of research are included in section VI.

\section{Previous Work on Reconstruction from Zero Crossings}

Zero crossing representation of signals is a special case of implicit sampling. Most of the work in this area has been in communication theory and has concentrated on onedimensional signals [5]. The two major existing results in implicit sampling of multidimensional signals deal with sig. nal representation in terms of zero crossings. The first one is by Rotem and Zeevi [16] and the other one is by Curtis, Oppenheim, and Lim [17], [1]. We will begin with a review of the former one.

Rotem and Zeevi's results are an extension of Logan's results to two dimensions. Logan's theorem (Theorem 1$)^{2}$ states that one-dimensional bandpass signals of less than one octave in bandwidth which have only real zeros of degree one in common with their Hilbert transform, are represented up to a constant by their zero crossings [5]. Rotem and Zeevi extended this result to two kinds of twodimensional signals: those which are bandpass in both dimensions (Theorem 2), and those which are bandpass in one dimension and lowpass in the other (Theorem 3). Theorem 2 basically states that $f(x, y)$, a real square-integrable bandpass function in both dimensions, can be uniquely specified from its zero crossings to within a scale factor provided there exist

- a $y_{0}$ such that the one-dimensional function of $x, f(x$, $y_{0}$ ) and its Hilbert transform have only real zeros of degree one in common;

- a sufficiently high density sampling set $\left\{x_{k}\right\}$ such that the one-dimensional functions in $y$ given by $f\left(x_{k}, y\right)$ have only real zeros of degree one with their Hilbert transforms.

The required density of the sampling set is a function of the bandpass frequencies of $f(x, y)$ along the $x$ direction. Since the one-dimensional functions $f\left(x_{k}, y\right)$ and $f\left(x, y_{0}\right)$ satisfy Logan's conditions, we can construct the functions along vertical lines $x=x_{k}$, scale them according to the function on the horizontal line $y_{0}$, and then, having a sampling set $\left\{x_{k}\right\}$, the value of $f(x, y)$ is determined (up to a constant) at every point in the plane.

Rotem and Zeevi's result for the case where the signal is lowpass in one dimension and bandpass in the other is formally given in Theorem 3 . It states that $f(x, y)$, a real, square-

${ }^{2}$ In the body of this paper only an interpretive informal description of the theorems is given. The formal statement of all theorems is given in Appendix A. A proof is included only for theorems which represent original contributions of this paper. 
integrable function which is bandpass in the $x$ direction and low pass in the $y$ direction, is uniquely specified up to a scale factor from its zero crossings provided there exist

- a sufficiently high density sampling set $\left\{y_{k}\right\}$ such that the one-dimensional functions in $x, f\left(x, y_{k}\right)$ and their Hilbert transforms have only real zeros of degree one in common;

- a straight line $S$ oriented in certain directions such that the one-dimensional signal along $S$ is integrable, and that has only real zeros of degree one in common with its Hilbert transform.

The required range of the angle of line $S$ with the $x$ axis depends on the spectral characteristics of $f(x, y)$, i.e., the frequencies above or below which the Fourier transform of $f(x, y)$ is zero. In addition, the density of the sampling set $\left\{y_{k}\right\}$ depends on the bandwidth of $f(x, y)$ along the $y$ direction. In this case the function is determined on horizontal lines $y_{k}$ and scaled on the diagonal S. Since $\left\{y_{k}\right\}$ is a sampling set, it can be used to determine the value for every point between the lines.

A few examples of reconstructions for these results are included in [16]. The major drawback of these theorems is that most two-dimensional signals encountered in practice, such as images, are lowpass in both dimensions. The result by Curtis et al. deals with periodic signals which are bandlimited in both dimensions and, in particular, can be lowpass. Furthermore, unlike Theorems 2 and 3 , the results are truly two-dimensional since they exploit the fact that zero crossing contours for two-dimensional signals contain infinitely many points.

The result by Curtis et al. is based on the theory of bivariate polynomials. A bivariate polynomial will be represented in the form

$$
p(w, z)=\sum_{i=0}^{N_{w}} \sum_{j=0}^{N_{z}} a(i, j) w^{i} z^{j}
$$

$\Pi_{\left(N_{w}, N_{z}\right)}$ is used to refer to the space of polynomials $p(w, z)$ with maximum degree $N_{w}$ in $w$ and $N_{z}$ in $z$. $\Pi_{R}$ is used to refer to the space of polynomials $p(w, z)$ for which the maximum degree of the one-dimensional $p(x, x)$ is $R$. The degree of $p(x, x)$ is also referred to as the total degree of $p(w, z)$. Clearly, polynomials in $\Pi_{\left(N_{w}, N_{z}\right)}$ are also in $\Pi_{\left(N_{w}+N_{z}\right)}$. The distinction between the spaces of polynomials is related to the fact that in the $i-j$ plane associated with the coefficients in (1), $\Pi_{\left(N_{w}, N_{z}\right)}$ is defined by the rectangular region of support $\left(N_{w}\right.$ $+1) \times\left(N_{z}+1\right)$, while $\Pi_{R}$ is defined by a triangular region of support. A bivariate polynomial is referred to as irreducible over complex numbers if it cannot be factored in polynomials of smaller degree with complex coefficients.

The formal description of the result by Curtis et al. is given by Theorem 5 . Theorem 5 states that a real two-dimensional doubly periodic bandlimited signal $f(x, y)$ is uniquely specified to within a scale factor from a finite number of samples of its zero crossings provided its associated bivariate polynomial (BLP) is irreducible. For a BLP signal of the form

$$
f(x, y)=\sum_{k_{x}=-N_{k}}^{N_{x}} \sum_{k_{y}=-N_{y}}^{N_{y}} F\left(k_{x}, k_{y}\right) \mathrm{e}^{\mathrm{i} 2 \pi\left(k_{x} x+k_{y} y\right)}
$$

with period 1 in $x$ and $y$ directions, and $\left(2 N_{x}+1\right) \times\left(2 N_{y}+\right.$ 1) region of support in the Fourier domain, the associated polynomial is defined as:

$$
\begin{aligned}
g(w, z) & =f(x, y) w^{N_{x}} z^{N_{y}} \\
& =\sum_{k_{x}=0}^{2 N_{x}} \sum_{k_{y}=0}^{2 N_{y}} F\left(k_{x}-N_{x}, k_{y}-N_{y}\right) w^{k_{x}} z^{k_{y}}
\end{aligned}
$$

where

$$
w=\mathrm{e}^{j 2 \pi x}, \quad z=\mathrm{e}^{j 2 \pi y} .
$$

In terms of the preceding definitions, Theorem 5 implies that $4\left(N_{x}+N_{y}\right)^{2}+1$ samples of the zero crossings of $f(x, y)$ are sufficient for its unique specification to within a scale factor provided $g(w, z)$ is irreducible over complex numbers. Since the set of reducible two-dimensional polynomials are of measure zero in the set of two-dimensional polynomials [13], most BLP signals encountered in practice are likely to have irreducible associated polynomials.

This theorem is a direct consequence of Bezout's theorem [40], [41] (Theorem 4), which provides an upper bound on the number of common zeros of bivariate polynomials. Specifically, it states that two bivariate polynomials of total degrees $R$ and $S$, which have no factors or order greater than zero, can have a maximum of $R S$ common finite zeros. Zakhor and Izraelivitz [18] (Theorem 6) have modified Bezout's theorem to derive a tighter bound on the number of finite common zeros of two polynomials whose coefficients have rectangular (rather than triangular) regions of support. Theorem 6 basically states that two relatively prime polynomials $p(x, y)$ and $q(x, y)$ whose coefficients have $\left(N_{x}+1\right)$ $\times\left(N_{y}+1\right)$ and $\left(M_{x}+1\right) \times\left(M_{y}+1\right)$ regions of support, respectively, cannot have more than $N_{x} M_{y}+N_{y} M_{x}$ common finite zeros. This result has been used to modify Theorem 5. Specifically, it implies that a BLP signal of the form given by (2) with an irreducible associated polynomial is uniquely specified up to a scale factor from $8 N_{x} N_{y}+1 \mathrm{sam}$ ples of its zero crossings. The modified version of Bezout's theorem will also be instrumental in deriving new results in Section III-A.2.

A possible algorithm for reconstruction based on Theorem 5 involves solving an overdetermined system of linear equations to find the Fourier coefficients of the signal under consideration. ${ }^{3}$ Examples of such reconstructions are included in [17], [1]. Potential applications of the result include recovery in recovery of images which have been corrupted by memoryless nonlinear distortions containing at least one monotone region, and the recovery of bandlimited continuous-tone images from halftones [15].

The experimental results in [1] indicate that the major drawback to reconstruction from zero crossings is the extreme sensitivity to the locations of the zero crossings. For instance, reconstruction of a BLP signal with a $31 \times 31$ region of support in the Fourier domain requires approximately 56 bits of accuracy for each coordinate of a zero crossing sample. This is an illustration of the fact that a zero crossing representation needs the minimum number of amplitude bits (i.e., 1) and a large number of position bits, in contrast to Nyquist sampling, which requires relatively few position bits and a large number of amplitude bits. In the next section, we propose semi-implicit and implicit sampling strategies for reconstruction from multiple-level crossings, in an attempt to bridge the gap between these two extremes.

${ }^{3}$ This is described in more detail in Section IV. 


\section{Theoretical Results}

In this section, we will derive two sampling strategies for reconstruction of multidimensional signals from multiplelevel threshold crossings. Our approach to this problem is similar to that in [1] in the sense that we represent BLP signals in terms of polynomials. The reasons for doing so are twofold. First, as indicated in (2)-(4), BLP signals can be written as polynomials via whose Fourier series expansion. Second, since reconstruction from multiple-level crossings is a special case of reconstruction from nonuniform samples, we hope to be able to use a variety of mathematical results on polynomial interpolation theory. Unfortunately, unlike the univariate case, interpolation with multivariate polynomials is a nontrivial task. Whereas $n$ arbitrary samples of a one-dimensional polynomial of degree $n-1$ are sufficient to find its coefficients, the analogous result in higher dimensions does not hold [19]-[22].

In this section we propose two ways to circumvent this difficulty. The first approach, described in Section III-A, involves imposing certain restrictions on the locations of the interpolation points in order to guarantee a unique solution to the resulting interpolation problem, and therefore represents semi-implicit sampling. The second approach, discussed in Section III-B, deals with implicit sampling based on conditionally regular interpolation methods. ${ }^{4}$

\section{A. Semi-Implicit Sampling}

Semi-implicit samples of a multidimensional signal are defined to be points whose coordinates are related to each other, or equivalently their locations are constrained. Our approach in this section is to constrain the location of the samples of the signal in such a way that unique recovery of its associated polynomial (or equivalently the signal itself) is guaranteed. This is a problem in bivariate polynomial interpolation theory which has been an active area of research for many years in the mathematics community [19], [24]-[29]. We begin with a brief review of some of the existing results in this field.

1) Review of Bivariate Polynomial Interpolation Theory: Bivariate polynomial interpolation can be done either in $\Pi_{R}$ the space of polynomials with total degree less than or equal to $R$, or in $\Pi_{\left(N_{w}, N_{z}\right)}$, the space of polynomials $p(w, z)$ with maximum degree $N_{w}$ in $w$ and $N_{z}$ in $z$. The most well-known result on interpolation in $\Pi_{\left(N_{w}, N_{z}\right)}$ deals with the case where the interpolation points are on a nonuniform rectangular grid [19], [24]-[26]. It states that the set of points

$$
\left\{\left(w_{i}, z_{j}\right) \mid i=0, \cdots, N_{w i} \quad j=0, \cdots, N_{z}\right\}
$$

uniquely specify the polynomial

$$
p(w, z)=\sum_{i=0}^{N_{w}} \sum_{j=0}^{N_{z}} a(i, j) w^{i} z^{j}
$$

There are serious limitations in applying this theorem to the problem of reconstruction from level crossings. This is

${ }^{4}$ An interpolation method is called "regular" if it is uniquely solvable for any selection of the points of interpolation. Conditionally regular interpolation methods are not solvable for all selections of points, but only for most of them [23]. For methods of this type, if one has a concrete problem and selects the interpolation points at random, it will be extremely unlikely that the problem will be unsolvable. because the available threshold contours are highly unlikely to pass through a nonuniform rectangular grid. A more general result on interpolation in $\Pi_{R}$ was derived by Gasca and Maeztu [27]. The formal description of their result is given by Theorem 7. It states that a polynomial in $\Pi_{R}$ with $(R+$ 1) $(R+2) / 2$ coefficients is uniquely specified from $(R+1)(R$ $+2) / 2$ of its samples distributed on lines $I_{0}, \cdots I_{R}$, provided there are $(2 i+1)$ points on the $i$ th line $e^{5}$. Different proofs of Gasca and Maeztu's result are included in [19], [27], and [28], and special cases of their theorem were proved earlier by Stenger [29] and Chung [24].

Theorem 7 can be applied to the problem of reconstruction from level crossings. For instance, suppose that we are interested in reconstructing a real BLP signal with $\left(2 N_{x}+\right.$ 1) $\times\left(2 N_{y}+1\right)$ region of support in the Fourier domain. The polynomial associated with this signal has total degree of $2 N_{x}+2 N_{y}$, and if we choose its $2 N_{x}+2 N_{y}+1$ sampling lines to be of the form

$$
z=e^{i 2 \pi \beta_{i}} w \quad 0 \leq i<2 N_{x}+2 N_{y}+1
$$

then, by (4), the corresponding lines in the $x-y$ domain will be of the form $y=x+\beta_{i}$. Theorem 7 guarantees that the sampling set consisting of $i$ samples on the ith sampling line in the $x-y$ domain uniquely specifies the BLP signal under consideration. Clearly, for the problem of reconstruction from level crossings, these samples must be chosen at the intersection of sampling lines and threshold contours.

From this example, we realize that for a signal of the form of (2), we need a total of $\left(N_{x}+N_{y}-1\right)\left(N_{x}+N_{y}-2\right) / 2 \mathrm{sam}-$ ples to guarantee its unique reconstruction. It can easily be shown that this number of required samples is at least twice the number of unknown Fourier coefficients. If the support regions of the Fourier coefficients of signals are rectangular rather than triangular, we can avoid oversampling by modifying Theorem 7 to apply to polynomials in $\Pi_{\left(N_{x}, N_{y}\right)}$ with the additional advantage that the locations of the interpolation points become considerably less restrictive.

2) New Results on Bivariate Interpolation Theory: As mentioned in Section II, Bezout's theorem (Theorem 4) deals with polynomials in $\Pi_{R}$. Specifically, it states that if two polynomials in $\Pi_{R}$ and $\Pi_{S}$ have no common factors, then they have at most $R S$ common zeros. The modified version of Bezout's theorem (Theorem 6) deals with polynomials in $\Pi_{\left(N_{w}, N_{z}\right)}$. It basically says that two relatively prime polynomials in $\Pi_{\left(N_{w}, N_{z}\right)}$ and $\Pi_{\left(M_{w}, M_{z}\right)}$ can not have more than $N_{w} M_{z}$ $+M_{w} N_{z}$ common finite zeros. An implication of this theorem is that if a polynomial $p(w, z) \in \Pi_{\left(N_{w}, N_{2}\right)}$ has more than $N_{w} M_{z}+M_{w} N_{z}$ common zeros with an irreducible polynomial $q(w, z) \in \Pi_{\left(M_{w}, M_{z}\right)}$, then $q(w, z)$ must be a factor of $p(w$, $z)$. We can use this to derive Theorem 8 , which is a more general version of Theorem 7 . Theorem 8 states that the bivariate polynomial of the form shown in (5) is uniquely reconstructible from samples on $n_{c}+1$ distinct irreducible curves provided there are a minimum of

$$
\begin{aligned}
S(i) \equiv & M_{z}^{(i)}\left(N_{w}-\sum_{k=0}^{i-1} M_{w}^{(k)}\right) \\
& +M_{w}^{(i)}\left(N_{z}-\sum_{k=0}^{i-1} M_{z}^{(k)}\right)+1 \quad 0 \leq i \leq n_{c}
\end{aligned}
$$

${ }^{5}$ The ordering of the lines can be drawn arbitrarily. 
samples on the ith curve. The maximum degrees of the $i$ th curve in $w$ and $z$ are defined to be $M_{w}^{(i)} M_{z}^{(i)}$, respectively. The number of required sampling curves, $n_{c}+1$, is defined to be an integer satisfying either of the following two conditions:

$$
\begin{aligned}
& N_{w}<\sum_{i=0}^{n_{c}} M_{w}^{(i)} \\
& N_{z}<\sum_{i=0}^{n_{c}} M_{z}^{(i)} .
\end{aligned}
$$

For the special case of Theorem 8 for which all the sampling curves are chosen to be lines passing through the origin, there is an alternative proof which provides a recursive way of computing the coefficients of the polynomial under consideration [30]. This proof is included in Appendix B. An example of the distribution of sampling points required by Theorem 8 for $N_{w}=N_{z}=2, M_{w}^{(0)}=M_{z}^{(0,1)}=1$ and $M_{w}^{(1)}=2$ is shown in Fig. 1(a). The irreducible curves in this example are of the form:

$$
\begin{aligned}
& C_{0}: w z=\alpha_{0} \\
& C_{1}: z^{2}=\alpha_{1} w .
\end{aligned}
$$

Unlike Theorem 7, Theorem 8 deals with polynomials in $\Pi_{\left(N_{m}, N_{z}\right)}$. It is also considerably more general than Theorem 7 in the sense that the interpolation points can be chosen on any irreducible curves (polynomials) rather than straight

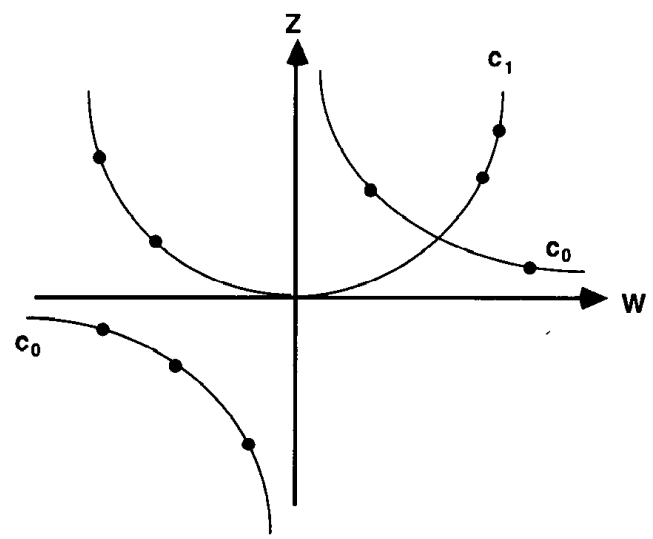

(a)

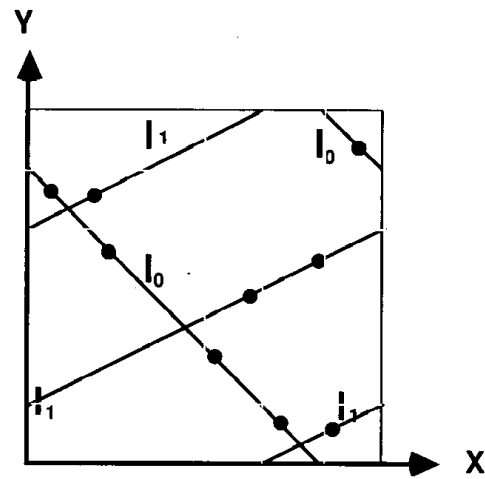

(b)

Fig. 1. Geometric distribution of sampling points. (a) Theorem (8) for $N_{w}=N_{z}=2$. (b) Corollary (1) for $N_{x}=N_{y}=1$. lines. ${ }^{6}$ In general, determining the irreducibility of polynomials is a nontrivial task [31]. Two classes of polynomials that are known to be irreducible and are particularly useful in deriving sampling strategies for multidimensional signals are of the form

$$
z^{M_{y}}=\alpha w^{M_{x}}, \quad z^{M_{y}} w^{M_{x}}=\alpha, \quad M_{x}>0, \quad M_{y}>0
$$

where $M_{y}$ and $M_{x}$ are relatively prime positive integers. Using the fact that $w$ and $z$ of (2) are related to $x$ and $y$, the signal coordinates, via (4), and letting $\alpha=\mathrm{e}^{j 2 \pi \beta}$, the curves in the $w-z$ plane given by (8) correspond to lines with positive or negative rational slope of the form

$$
\begin{gathered}
M_{y} y=\beta+M_{x} x, \quad M_{y} y+M_{x} x=\beta, \\
M_{x}>0, M_{y}>0
\end{gathered}
$$

in the $x-y$ plane. We can use this fact, together with Theorem 8 , to define a sampling strategy for BLP signals in which the samples are chosen on lines of rational slope. This sampling scheme is described in Corollary 2 in Appendix $B$, and states that the two-dimensional BLP signal of the form shown in (2) is uniquely reconstructible from samples on $N_{l}+1$ distinct lines with rational slope provided there are a minimum of

$$
S(i)=M_{y}^{(i)}\left(2 N_{x}-\sum_{k=0}^{i-1} M_{x}^{(k)}\right)+M_{x}^{(i)}\left(2 N_{y}-\sum_{k=0}^{i-1} M_{y}^{(k)}\right)+1
$$

samples on the $i$ th line. The ith line is defined to be of the forms

$$
M_{y}^{(i)} y=M_{x}^{(i)} x, \beta_{i}, \quad M_{y}^{(i)} y+M_{x}^{(i)} x=\beta_{i}, \quad M_{x}^{(i)}, M_{y}^{(i)}>0
$$

and the number of required sampling lines, $N_{t}+1$, is defined to be an integer satisfying either of the following two conditions:

$$
\begin{aligned}
& 2 N_{x}<\sum_{i=0}^{N_{t}} M_{x}^{(i)} \\
& 2 N_{y}<\sum_{i=0}^{N_{i}} M_{y}^{(i)} .
\end{aligned}
$$

Whereas Theorem 7 can be used to reconstruct multidimensional BLP signals from their samples on lines of slope one, Corollary 2 to Theorem 8 requires samples on lines of positive or negative rational slope. An example of the geometric distribution of the sampling points required by this corollary for $N_{x}=N_{y}=1$ is shown in Fig. 1(b). The sampling lines in this example are of the form

$$
\begin{aligned}
& l_{0}: y+x=\beta_{0} \\
& l_{1}: 2 y=x+\beta_{1} .
\end{aligned}
$$

Note that the curves of Fig. 1(a) in $w-z$ domain correspond to lines of Fig. 1(b) in the $x-y$ domain. In addition, since we are dealing with periodic signals in the $x-y$ domain, lines of rational slope "wrap around" or extend modulo 1 ' in $x$ and $y$ throughout the signal under consideration. This is illustrated in Fig. 1(b), where lines $I_{0}$ and $I_{1}$, with slopes -1 and $1 / 2$, are wrapped around once and twice, respectively.

To apply Corollary 2 to the problem of reconstruction from multiple-level crossings, we choose the intersections

${ }^{6}$ Note that straight lines are a special case of irreducible polynomials.

${ }^{7}$ We have assumed the period to be 1 in both $x$ and $y$ directions. 
of sampling lines and the threshold contours as the interpolation points. One drawback of such a reconstruction strategy is that, in general, there is no guarantee that we will obtain enough intersections to satisfy the distribution required by Corollary 2. Of course, we can propose various guidelines for choosing the slope and position of the sampling lines in an "optimal" fashion so that the number of intersections is maximized [30]. However, the problem still remains that for a small number of thresholds there might not be any set of sampling lines which satisfy the theoretical requirements. This is the main motivation for deriving the implicit sampling scheme of Section III-B.

Before we end this section, let us make a few observations on the more general problem of reconstruction from crossings with arbitrary functions. For certain class of functions such as sinusoids, the number of intersections of sampling lines with function crossings of the signal becomes predictable. As an example, consider the eye picture shown in Fig. 2(a) and its sinusoid crossings with the function

$$
h(x, y)=A+B \cos (2 \pi(p x+q y))
$$

shown in Fig. 2(b) for $p=q=1$. As seen, $h(x, y)$ assumes its maximum value, $A+B$, and minimum value, $A-B$, on equidistant lines of slope $-(p / q)$. If we impose the condition

$$
\begin{aligned}
A-B & =[h(x, y)]_{\min }<f(x, y)<[h(x, y)]_{\max } \\
& =A+B, \quad \forall(x, y)
\end{aligned}
$$

on the amplitude of the signal $f(x, y)$, then between every two neighboring parallel lines where $h(x, y)$ assumes its minimum and maximum values, there exists a contour of crossings of $h(x, y)$ with $f(x, y)$. Under these conditions, the number of intersections of a line with rational slope $n / m$ with the sinusoid crossings is guaranteed to be $2\lceil\mathrm{pm}+$ $q n\rceil$, where $\lceil a\rceil$ is defined to be the smallest integer greater than a. As shown in Fig. 2(b), each intersection point lies between the two lines at which $h(x, y)$ assumes its maximum and minimum values. This more or less even distribution of crossings across the image results in a well-posed reconstruction problem, which can be successfully solved via reasonably efficient reconstruction algorithms [30]. In addition, reconstruction of signals from their intersections with sinusoid crossings can be applied to recovery of continuous-tone images from halftones [15].

\section{B. Implicit Approach to Reconstruction}

In this section we develop an implicit sampling strategy based on algebraic geometric concepts. Unlike the semiimplicit method, this approach can be used for reconstruction from an arbitrarily small number of level crossings. However, since it corresponds to conditionally regular interpolation, it is not guaranteed to be solvable for all selections of points on the level-crossings contours, but most of them.

Consider a two-dimensional real BLP signal $f(x, y)$ whose Fourier series expansion is given by (2). For simplicity we assume $F\left(k_{x}, k_{y}\right)$ to have a square region of support of size $(2 N+1) \times(2 N+1)$. Our results can be easily modified for the case when $F\left(k_{x}, k_{y}\right)$ has a rectangular region of support. Since $f(x, y)$ is real, its Fourier series coefficients are conjugate symmetric. That is,

$$
F\left(k_{x}, k_{y}\right)=F^{*}\left(-k_{x},-k_{y}\right) \quad\left|k_{x}\right|,\left|k_{y}\right|<N .
$$

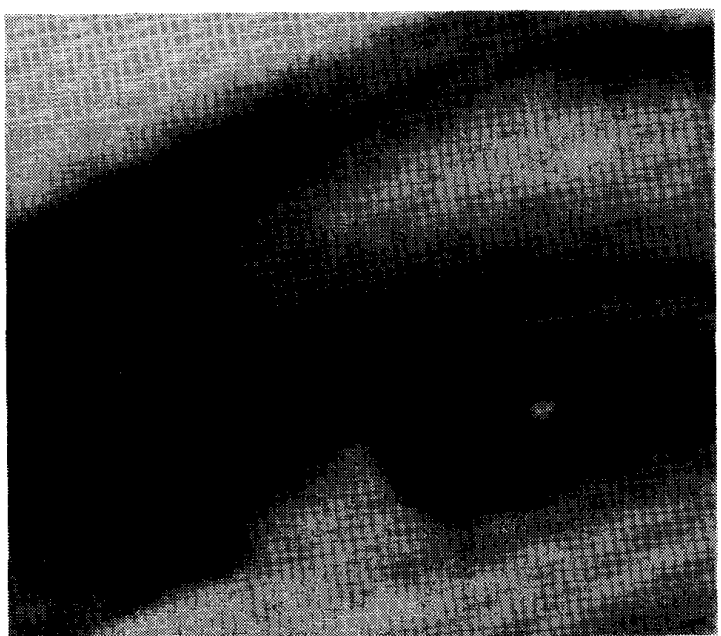

(a)

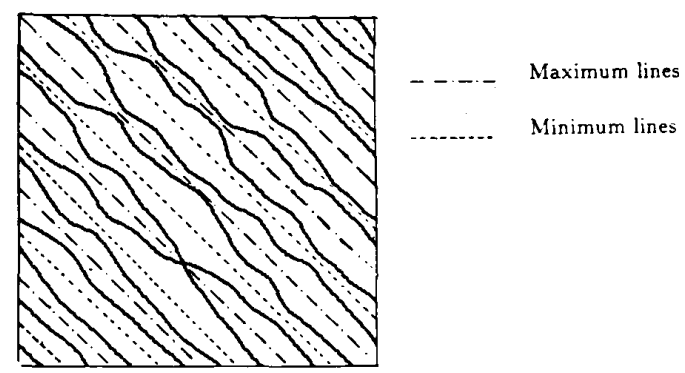

(b)

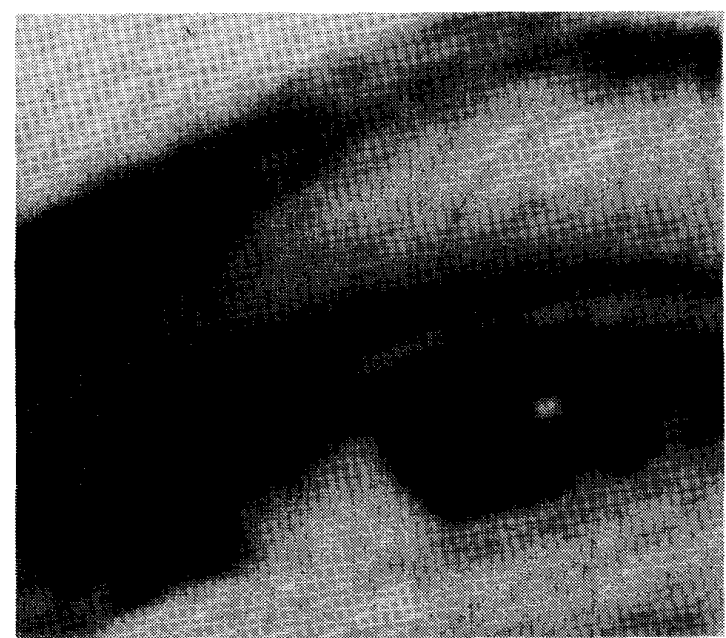

(c)

Fig. 2. (a) Original $256 \times 256$ eye picture with $31 \times 31$ region of support in Fourier domain. (b) Sinusoid crossings of eye picture: the lines at which the sinusoid assumes its maximum and minimum values are also shown. (c) Reconstructed version of eye picture via linear least-squares approach and $Q R$ decomposition from intersections of eight level crossings, shown in (a), with 15 lines of sloped 1, 2 $\pm \frac{1}{2}, \pm 3, \pm \frac{1}{3}, \pm 4, \pm \frac{1}{4}, 5$. 
Using this relationship, $f(x, y)$ can be rewritten as

$$
\begin{aligned}
f(x, y)= & \operatorname{Re}[F(0,0)]+2\left\{\sum_{k_{y}=1}^{N} \operatorname{Re}\left[F\left(0, k_{y}\right)\right] \cos \left(2 \pi k_{y} y\right)\right. \\
& -\operatorname{Im}\left[F\left(0, k_{y}\right)\right] \sin \left(2 \pi k_{y} y\right) \\
& +\sum_{k_{x}=1}^{N} \sum_{k_{y}=-N}^{N} \operatorname{Re}\left[F\left(k_{x}, k_{y}\right)\right] \cos \left[2 \pi\left(k_{x} x+k_{y} y\right)\right] \\
& \left.-\operatorname{Im}\left[F\left(k_{x}, k_{y}\right)\right] \sin \left[2 \pi\left(k_{x} x+k_{y} y\right)\right]\right\} .
\end{aligned}
$$

Thus $f(x, y)$ can be uniquely specified with $(2 N+1)^{2}$ real numbers which represent the real and imaginary part of its Fourier series coefficients. Therefore unique reconstructibility of $f(x, y)$ from $(2 N+1)^{2}$ samples of it, given by

$$
\left(x, y_{i}\right) i=1, \cdots,(2 N+1)^{2}
$$

is equivalent to nonsingularity or full rank of the $(2 N+1)^{2}$ $\times(2 N+1)^{2}$ matrix whose ith row, $r_{i}$, is given by

$$
r_{i}^{T}=\left[\begin{array}{c}
1 \\
\cos \left(2 \pi y_{i}\right) \\
\cos \left(4 \pi y_{i}\right) \\
\cdots \\
\cos \left(2 N \pi y_{i}\right) \\
\sin \left(2 \pi y_{i}\right) \\
\cdots \\
\sin \left(2 N \pi y_{i}\right) \\
\cos \left[2 \pi\left(x_{i}-N y_{i}\right)\right] \\
\cdots \\
\cos \left[2 \pi\left(N x_{i}+N y_{i}\right] .\right. \\
\sin \left[2 \pi\left(x_{i}-N y_{i}\right)\right] \\
\cdots \\
\sin \left[2 \pi\left(N x_{i}+N y_{i}\right)\right]
\end{array}\right]
$$

We will refer to this matrix as being associated with the points $\left(x_{i}, y_{i}\right), i=1, \cdots,(2 N+1)^{2}$, and denote its determinant by the function $d\left(x_{1}, y_{1}, \cdots, x_{(2 N+1)^{2}}, y_{\left.(2 N+1)^{2}\right)}\right.$. Let us define the sets $A_{\gamma}$ and $A_{\delta}$ as $\gamma$ and $\delta$ level crossings of $f(x, y)$ :

$$
\begin{aligned}
& A_{\gamma}(C)=\left\{(x, y) \in C^{2} \mid f(x, y)=\gamma\right\} \\
& A_{\gamma}(R)=\left\{(x, y) \in R^{2} \mid f(x, y)=\gamma\right\}=A_{\gamma}(C) \cap R^{2} \\
& A_{\delta}(C)=\left\{(x, y) \in C^{2} \mid f(x, y)=\delta\right\} \\
& A_{\delta}(R)=\left\{(x, y) \in R^{2} \mid f(x, y)=\delta\right\}=A_{\delta}(C) \cap R^{2}
\end{aligned}
$$

where $C^{2}$ and $R^{2}$ are the two-dimensional fields of complex and real numbers, respectively. Furthermore, let $B(R) \subset$ $R^{2(2 N+1)^{2}}$ denote the cross product

$$
B(R)=A_{\gamma}^{k}(R) \times A_{\delta}^{(2 N+1)^{2}-k}(R)
$$

where $A_{\gamma}^{k}(R)$ is $k$ times the cross product of $A_{\gamma}(R)$ with itself, and $A_{\delta}^{(2 N+1)^{2}-k}(R)$ is $(2 N+1)^{2}-k$ times the cross product of $A_{\delta}(R)$ with itself.

To show that almost any $0<k<(2 N+1)^{2}$ points from real $\gamma$ level crossings (i.e., $\left.A_{\gamma}(R)\right)$ and $(2 N+1)^{2}-k$ points from real $\delta$ level crossings (i.e., $A_{\delta}(R)$ ) are sufficient for unique specification of $f(x, y)$, we must find conditions under which real zeros of the function $d\left(x_{1}, y_{1}, \cdots, x_{(2 N+1)^{2}}, y_{(2 N+1)^{2}}\right)$ become of measure zero in the set $B(R) \subset R^{2(2 N+1)^{2}}$. To formulate this in an algebraic geometric framework, we change variables to express trigonometric functions such as $d$ as algebraic polynomials. ${ }^{8}$ To this end, let

$$
\begin{aligned}
& \sin (2 \pi x)=w, \quad \cos (2 \pi x)=z, \\
& \sin (2 \pi y)=u, \quad \cos (2 \pi y)=v
\end{aligned}
$$

so that

$$
f(x, y)=\tilde{f}(w, z, u, v)
$$

$d\left(x_{1}, y_{1}, \cdots, x_{(2 N+1)^{2}}, y_{(2 N+1)^{2}}\right.$

$=\tilde{d}\left(w_{1}, z_{1}, u_{1}, v_{1}, \cdots, w_{(2 N+1)^{2}}, z_{(2 N+1)^{2}}, u_{(2 N+1)^{2}}, v_{\left.(2 N+1)^{2}\right)}\right.$

$$
\begin{aligned}
& \tilde{A}_{\gamma}(C)=\left\{\begin{array}{r}
(w, z, u, v) \in C^{4} \mid \tilde{f}(w, z, u, v)=\gamma \\
w^{2}+z^{2}=1 \\
u^{2}+v^{2}=1
\end{array}\right\} \\
& \tilde{A}_{\delta}(C)=\left\{\begin{array}{r}
(w, z, u, v) \in C^{4} \mid \tilde{f}(w, z, u, v)=\delta \\
w^{2}+z^{2}=1 \\
u^{2}+v^{2}=1
\end{array}\right\} \\
& \tilde{A}_{\delta}(R)=\bar{A}_{\delta}(C) \cap R^{4}, \bar{A}_{\gamma}(R)=\tilde{A}_{\gamma}(C) \cap R^{4} \\
& \tilde{B}(C)=\tilde{A}_{\gamma}^{k}(C) \times \tilde{A}_{\delta}^{(2 N+1)^{2}-k}(C) \\
& \tilde{B}(R)=\tilde{B}(C) \cap R^{2(2 N+1)^{2}}=\tilde{A}_{\gamma}^{k}(R) \times \tilde{A}_{\delta}^{(2 N+1)^{2}-k}(R) .
\end{aligned}
$$

Since there is a one-to-one correspondence between elements of the sets $A_{\gamma}$ and $\tilde{A}_{\gamma}, A_{\delta}$ and $\tilde{A}_{\delta}$, and $B$ and $\bar{B}$, we must find conditions under which real zeros of $\tilde{d}\left(w_{1}, z_{1}, \cdots\right.$, $\left.u_{(2 N+1)^{2}}, v_{(2 N+1)^{2}}\right)$ have measure zero in $\tilde{B}(R)$. Unlike $A_{\gamma}, A_{\delta \prime}$ and $B$, the sets $\bar{A}_{\gamma}(R)$ and $\bar{A}_{\delta}(R)$ are algebraic sets in $R^{4}$, and $\tilde{B}(R)$ is an algebraic set in $R^{4(2 N+1)^{2}}$. An algebraic set is one which can be written as common zeros of a set of polynomials. Specifically, if $A^{n}$ denotes the $n$ time Cartesian product of a field $A$ with itself, then $X \subset A^{n}$ is an algebraic set in $A^{n}$ provided there exists a set of polynomials $S$, with coefficients in $A$, such that the set of their common zeros $V(S)$ is identical to $X$ [32]. An algebraic set $V$ is reducible if $V=V_{1} \cap V_{2}$, where $V_{1}$ and $V_{2}$ are algebraic sets in $A^{n}$, distinct from each other and $V . V_{1}$ and $V_{2}$ are referred to as components of $V$. If $V$ is not reducible, it is irreducible. Given an irreducible algebraic set $V$ in $A^{n}$, the zeros of a polynomial with coefficients in $A$ are either of measure zero in $V$ or contain all the points in $V$. Since we want to show that zeros of $\bar{d}$ are of measure zero in $\tilde{B}(R)$, we must find conditions under which $\tilde{B}(R)$ becomes irreducible. These conditions, which are included in Theorem 9, can be summarized in the following way:

1) $\tilde{A}_{\gamma}(R)$ and $\bar{A}_{\delta}(R)$ have maximal topological dimensions';

2) the polynomials associated with $\gamma$ and $\delta$ level crossings are irreducible over complex numbers.

In effect, Theorem 9 provides conditions under which twodimensional BLP signals with $(2 N+1) \times(2 N+1)$ region of

${ }^{8}$ This is because algebraic geometry deals only with zeros of algebraic polynomials rather than trigonometric ones.

${ }^{9}$ Definition of topological dimension is given in Appendix C. 
support in the Fourier domain can be uniquely specified from almost any $0<k<(2 N+1)^{2}$ points from their $\gamma$ crossings and $(2 N+1)^{2}-k$ points from their $\delta$ crossings. It is natural to question the strictness of these conditions in practical situations. The first condition in Theorem 9 requires $\bar{A}_{\gamma}(R)$ and $\bar{A}_{\delta}(R)$ to have maximal topological dimensions. Since the complex topological dimensions of both $\bar{A}_{\gamma}(C)$ and $\bar{A}_{\delta}(C)$ are 1 , maximal dimensions for both $\bar{A}_{\gamma}(R)$ and $\bar{A}_{\delta}(R)$ are 1 . This implies that $\gamma$ and $\delta$ level crossings of $f(x, y)$ must contain at least a curve rather than isolated points. By proper choice of $\gamma$ and $\delta$, this condition can be easily satisfied in practice. This is also true of the second condition of the theorem in the sense that the set of reducible polynomials have been shown to be of measure zero in the set of all bivariate polynomials with conjugate symmetric coefficients [33], [34].

Theorem 9 can be extended to recovery of multidimensional signals from more than two threshold crossings. Specifically, we can show that given $m$ distinct thresholds $t_{1}$, $\cdots, t_{m}$, almost any distribution of $(2 N+1)^{2}$ points among the thresholds will result in unique reconstruction of the signal under consideration, provided the topological dimensions of the level crossings are maximal and their associated polynomials are irreducible. Furthermore, this theorem can be extended to reconstruction from crossings with BLP functions whose bandwidth lies within the bandwidth of the signal under consideration. Finally, Theorem 9 implies that almost any $(2 N+1)^{2}$ samples of one level crossings of a signal with $(2 N+1) \times(2 N+1)$ region of support in the Fourier domain is sufficient for its unique reconstruction to within a scale factor, provided the polynomial associated with the signal is irreducible. This is in contrast with $\left(16 N^{2}+1\right)$ samples required by Theorem 5 and discussed in Section II.

\section{Reconstruction Algorithms}

In this section, we propose a number of algorithms for reconstruction of signals from implicit or semi-implicit samples of their multiple-level threshold crossings. Our first approach involves solving linear systems of equations, and the second one is an iterative approach based on the theory of projection on convex sets (POCS) [35]. In both cases, we exploit the theoretical results of Section III to ensure that each reconstruction algorithm will almost always have a unique solution.

\section{A. Linear Least-Squares Approach}

The most straightforward approach to reconstruction from one- or multiple-level crossings is to solve a linear system of equations. To reconstruct a $(2 N+1) \times(2 N+1)$ BLP signal from $N_{t}$ thresholds, $N_{s} \geq(2 N+1)^{2}$ semi-implicit or implicit samples of the level crossings can be used to determine the Fourier coefficients by solving the (possibly overdetermined) linear system of equations given by

$$
\begin{gathered}
\sum_{k_{x}=-N}^{N} \sum_{k_{y}=-N}^{N} F\left(k_{x}, k_{y}\right) e^{j 2 \pi\left(k_{x} x_{j}+k_{y} y_{j}\right)}=t_{i r} \\
1 \leq i \leq N_{t}, \quad 1 \leq j \leq M .
\end{gathered}
$$

It is important to emphasize that, in either case, we are not merely solving $N_{s} \geq(2 N+1)^{2}$ equations in $(2 N+1)^{2}$ unknowns. If $\left(x_{j}, y_{j}\right)$ are semi-implicit samples chosen on lines of rational slope, their distribution must satisfy the conditions of Theorem 6; otherwise the system of equations might become singular, resulting in non-unique solutions. Similarly, if $\left(x_{i}, y_{i}\right)$ are implicit samples chosen on threshold contours of the signal under consideration, the conditions of Theorem 9 must be satisfied to be almost always guaranteed of the nonsingularity of the preceding system of equations.

Fig. 2(c) shows the reconstructed versions of the eye picture, from the intersections of 13 sampling lines of slopes $1,2, \pm \frac{1}{2}, \pm 3, \pm \frac{1}{3}, \pm 4, \pm \frac{1}{4}, 5$ with eight level crossings, corresponding to semi-implicit sampling. Robustness of the linear least-squares approach depends on a number of factors, including the number of reconstruction samples and the actual algorithm used to solve the system of equations. For the example shown in Fig. 2(c), the number of reconstruction samples was 1548 , and QR decomposition was used to solve the equations. The most stable linear leastsquares algorithms, such as QR decomposition, are computation- and storage-intensive. In general, the storage requirement of these is proportional to $N^{4}$ for an $N \times N$ signal. ${ }^{10}$ To overcome the storage problem, we propose iterative algorithms for semi-implicit and implicit sampling strategies.

\section{B. Iterative Algorithms Based on POCS}

In this section we propose two iterative algorithms for reconstruction of multidimensional signals from semiimplicit and implicit samples. The basic idea behind these algorithms is to iterate between the space and frequency domains by successively imposing the appropriate constraints in each domain: the bandlimitedness of the signal constitutes the frequency domain constraint, and the space domain constraint is derived from the quantized semiimplicit or implicit samples. The convergence of these algorithms is based on the theory of POCS, while the uniqueness of the solutions are guaranteed by the theoretical results of Section III.

We begin with the iterative algorithm for implicit sampling.

1) The Iterative Algorithm for Implicit Sampling: Consider a BLP signal with $(2 N+1) \times(2 N+1)$ region of support in the Fourier domain and an $M \times M$ grid, where $M>2 N$ +1 as indicated in Fig. 3 (c). We assume $N_{t}$ thresholds $t_{1}<$ $t_{2}<\cdots<t_{N_{t}}$ with the minimum signal value less than $t_{1}$ and the maximum greater than $t_{N_{1}}$, so that each threshold crossing is a contour. From the threshold crossing contours we can associate with each gridpoint an upper and lower amplitude value. Specifically, with $t_{y}\left(n_{x}, n_{y}\right)$ and $t_{u}\left(n_{x}, n_{y}\right)$ denoting the lower and upper bound for the $\left(n_{x}, n_{y}\right)$ th point on the grid,

$$
\begin{aligned}
t_{i}\left(n_{x}, n_{y}\right) \leq f\left(\frac{n_{x}}{M}, \frac{n_{y}}{M}\right) & \leq t_{u}\left(n_{x}, n_{y}\right) \\
n_{x}, n_{y} & =0, \cdots, M-1 .
\end{aligned}
$$

\footnotetext{
${ }^{10}$ Iterative algorithms, such as conjugate gradient, which do not need to store the matrix explicitly, converge very slowly. However they can only be successfully applied to well-conditioned problems such as reconstruction from sinusoid crossings. In addition, the recursive approach described in Appendix B is not nearly as storage-intensive as the least-squares approach. However, in general, it does not result in robust reconstruction.
} 

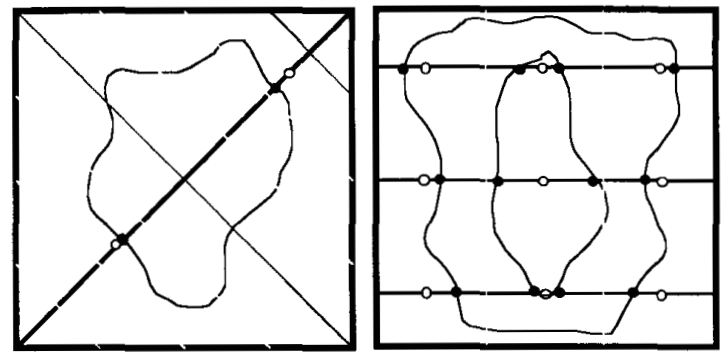

- Non-quantized samples

- Quantized samples

(a)

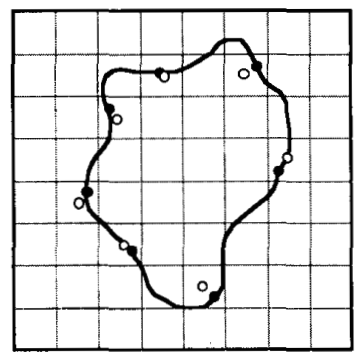

- Non-quantized samples

- Quantized samples

(c)

(d)

Fig. 3. (a) Derivation of space-domain constraint for iterative algorithm of semi-implicit sampling. (b) If we have $N$ equidistant horizontal or vertical sampling lines, together with $N$ equally spaced samples on each line, resulting semiimplicit sampling set corresponds to amplitude quantized Nyquist samples. (c) Derivation of space-domain constraint for iterative algorithm of implicit sampling. (d) Amplitudequantized samples derived from quantized threshold contours by two-dimensional iterative algorithm are identical to amplitude quantized Nyquist samples.

The steps of the algorithm are as follows.

1) Let $f^{(l)}\left(n_{x} / M, n_{y} / M\right)$ denote the value of $f$ in the $/$ th iteration at the point $\left(n_{x} / M, n_{y} / M\right)$. Take the $M \times M$ discrete Fourier transform (DFT) of $f^{(\prime)}\left(n_{x} / M, n_{y} / M\right)$ to get $F^{(\prime)}\left(k_{x}, k_{y}\right)$.

2) Impose the bandlimited constraint:

$$
\tilde{F}^{(l+1)}\left(k_{x}, k_{y}\right)= \begin{cases}\tilde{F}^{(l)}\left(k_{x}, k_{y}\right), & 0 \leq k_{x}, k_{y} \leq N, \\ & M-N \leq k_{x}, k_{y}<M \\ 0, & \text { elsewhere. }\end{cases}
$$

3) Apply the operator $T_{1}=1+\lambda_{1}\left(P_{1}-1\right)$ to $f^{(I)}\left(n_{x} / M, n_{y} l\right.$ $M)$ to get

$$
\begin{aligned}
f^{\prime(l+1)}\left(\frac{n_{x}}{M}, \frac{n_{y}}{M}\right) \equiv & f^{(l)}\left(\frac{n_{x}}{M}, \frac{n_{y}}{M}\right) \\
& +\lambda_{1}\left[\tilde{f}^{(l+1)}\left(\frac{n_{x}}{M}, \frac{n_{y}}{M}\right)-f^{(l)}\left(\frac{n_{x}}{M}, \frac{n_{y}}{M}\right)\right] .
\end{aligned}
$$

$P_{1}$ is the bandlimiting operator of step (3) and $\tilde{f}^{(I+1)}\left(n_{x} I\right.$ $\left.M, n_{y} / M\right)$ is the inverse DFT of $\tilde{F}^{(l+1)}\left(k_{x}, k_{y}\right)$.
4) Impose the space domain constraint by projecting $f^{\prime(l+1)}$ onto $\breve{f}^{(l+1)}$ via the operator $P_{2}$ :

$$
\begin{aligned}
\breve{f}^{(l+1)}\left(\frac{n_{x}}{M}, \frac{n_{y}}{M}\right) \\
=P_{2}\left[f^{\prime(l+1)}\left(\frac{n_{x}}{M}, \frac{n_{y}}{M}\right)\right] \\
f^{\prime(l+1)}\left(\frac{n_{x}}{M}, \frac{n_{y}}{M}\right), \\
\equiv \quad t_{i}\left(n_{x}, n_{y}\right) \leq f^{\prime(l+1)}\left(\frac{n_{x}}{M^{\prime}}, \frac{n_{y}}{M}\right) \leq t_{u}\left(n_{x}, n_{y}\right) \\
f^{\prime(l+1)}\left(\frac{n_{x}}{M}, \frac{n_{y}}{M}\right)<t_{l}\left(n_{x}, n_{y}\right) \\
t_{u}\left(n_{x}, n_{y}\right), \\
f^{\prime(l+1)}\left(\frac{n_{x}}{M}, \frac{n_{y}}{M}\right)>t_{u}\left(n_{x}, n_{y}\right) .
\end{aligned}
$$

5) Apply the operator $T_{2} \equiv 1+\lambda_{2}\left(P_{2}-1\right)$ to $\check{f}^{(i+1)}$ :

$$
\begin{aligned}
f^{(l+1)}\left(\frac{n_{x}}{M}, \frac{n_{y}}{M}\right) \\
=f^{\prime(l+1)}\left(\frac{n_{x}}{M}, \frac{n_{y}}{M}\right) \\
\quad+\lambda_{2}\left[\breve{f}^{(l+1)}\left(\frac{n_{x}}{M}, \frac{n_{y}}{M}\right)-f^{\prime(l+1)}\left(\frac{n_{x}}{M}, \frac{n_{y}}{M}\right)\right) .
\end{aligned}
$$

6) If all the nodes of the $M \times M$ grid satisfy the space domain constraint, we are done. Otherwise repeat steps (2)-(7).

We can use the theory of POCS [35] to establish the convergence of this algorithm. The formal description of this theory is given by Theorem 10 . The theorem specifies a way of finding an element in $C_{0}$, the intersection of the sets $C_{1}$, $\cdots, C_{m}$ which are defined to be convex, closed subsets of a Hilbert space, $H$. It basically states that successive application of the operator

$$
T=T_{m} T_{m-1} \cdots T_{1}
$$

to any element in $H$ converges to an element in $C_{0}$ provided

$$
T_{i}=1+\lambda_{i}\left(P_{i}-1\right) \quad 0<\lambda_{i}<2, \quad 1 \leq i \leq m
$$

and $P_{i}$ is a projection operator onto $C_{i}$. The convergence is strong if either $H$ is finite-dimensional or if one of the subsets, e.g., $C_{r}$ is finite-dimensional and $\lambda_{r}=1$.

The iterative algorithm is essentially a special case of this theorem, where the Hilbert space $H$ is the finite-dimensional space of all $M \times M$-point real two-dimensional sequences and the inner product between two $M \times M$-point sequences $x_{1}\left(n_{x}, n_{y}\right)$ and $x_{2}\left(n_{x}, n_{y}\right)$ is defined to be

$$
\left\langle x_{1}, x_{2}\right\rangle=\sum_{n_{x}=0}^{M-1} \sum_{n_{y}=0}^{M-1} x_{1}\left(n_{x}, n_{y}\right) x_{2}\left(n_{x}, n_{y}\right)
$$

The convex closed subsets of $H$ are $C_{1}$ and $C_{2} . C_{1}$ is the set of all $M \times M$-point real bandlimited sequences whose DFT is zero in the range $N<k_{x}, k_{y}<M-N$, and $C_{2}$ is the set of all $M \times M$-point real sequences, which satisfy the space 
domain constraint derived from the level crossing information as described in the first step of the algorithm. $P_{1}$ is the bandlimiting operator which projects onto the set $C_{1}$, and $P_{2}$ is the projection operator which imposes the space domain constraint and projects onto $C_{2}$. Thus the iterative algorithm consists of successive applications of the operator $T=T_{1} T_{2}$ to an arbitrary initial condition, and by Theorem 10 it converges strongly to an element in the set $C_{0} \equiv$ $C_{1} \cap C_{2}$, which in this case is the set of $M \times M$-point real sequences satisfying both the space and frequency domain constraints. As long as $M$ is finite, there might be many sequences satisfying both constraints. However, in the limit as $M \rightarrow \infty$, Theorem 9 implies that the set $C_{0}$ will almost always contain exactly one sequence. Under these circumstances, $H$ will not be finite-dimensional and strong convergence is no longer guaranteed. However, by Theorem 10 , as long as at least one of the convex closed subsets, namely $C_{1}$, is finite-dimensional, strong convergence is guaranteed provided $\lambda_{1}=1$. In general, we can control the convergence rate of the algorithm by proper choice of the relaxation parameters $\lambda_{1}$ and $\lambda_{2}$. The simplest such technique is overrelaxation, which consists of setting $1<\lambda<$ $2^{11}$. Values of $\lambda$ outside the interval $(0,2]$ disrupt convergence.

Fig. 4(a) shows the reconstructed version of the eye picture from 4, 6, and 8 thresholds, using the iterative algorithm for different values of $M$. The relaxation parameters for all the reconstructions were chosen to be $\lambda_{1}=1, \lambda_{2}=$ 1.75. The initial guess was chosen to be

$$
\begin{aligned}
f^{(0)}\left(\frac{n_{x}}{M}, \frac{n_{y}}{M}\right) & =\frac{t_{(}\left(n_{x}, n_{y}\right)+t_{u}\left(n_{x}, n_{y}\right)}{2}, \quad n_{x}, n_{y} \\
& =0, \cdots, M-1
\end{aligned}
$$

As shown, increasing the number of thresholds improves the quality of reconstruction in a more substantial way than increasing $M$. Since the iterative algorithm is considerably less storage-intensive than the least-squares approach, it is capable of reconstructing larger images. An example of such a reconstruction is shown in Fig. 5(b), where vegas picture of Fig. 5(a) with $127 \times 127$ Fourier region of support is recovered from eight thresholds. The relaxation parameters were $\lambda_{1}=1$ and $\lambda_{2}=1.75$, and the grid size was $M=$ 512. In the next section, we describe the iterative algorithm for semi-implicit sampling, and in Section V, quantization characteristics of the iterative algorithms will be discussed.

3) Iterative Algorithm for Semi-Implicit Sampling: The iterative algorithm for semi-implicit sampling reconstructs the one-dimensional signals associated with the sampling lines of a $2 \mathrm{D}$ signal by imposing constraints in frequency and space domains successively. The 10 signals are then used to recover the $2 \mathrm{D}$ signal under consideration. Thus the iterative algorithm is part of a "line by line" reconstruction approach. This approach exploits the fact that signals defined along lines of rational slopes of 2D BLP signal, are also bandlimited and periodic, ${ }^{12}$ and by Fourier series uniqueness, can be specified by a finite number of their

${ }^{n}$ We speak of underrelaxation when $0<\lambda<1$. With $\lambda=2$, we have another special case, referred to by Motzkin and Schoenberg [36] as the "reflection method."

${ }^{12}$ The fact that the one-dimensional signal associated with a sampling line with rational slope is bandlimited and periodic was originally shown by Mersereau [37]. samples. For instance, the number of Fourier harmonics of the signal associated with a sampling line of slope $n / m$ for a signal with $(2 N+1) \times(2 N+1)$ support in the Fourier domain is $2 N(m+n)+1$, and therefore $2 N(m+n)+1$ samples of it are sufficient for its unique recovery.

Before describing the iterative algorithm, we make three comments. First, the iterative algorithm is one of many ways to recover the one-dimensional signals associated with the sampling lines. For instance, one could solve a linear system of equations (possibly overdetermined) to determine their Fourier coefficients. ${ }^{13}$ Second, the sampling requirements of line-by-line reconstruction are, in general, more stringent than those of Corollary 2. For instance, if all the sampling lines are chosen to be of slope $n / m$, line-by-line reconstruction requires $2 N(m+n)+1$ samples on each line, whereas Corollary 2 only requires one line with as many points as $2 \mathrm{~N}(m+n)+1$. Third, there are many ways to recover the two-dimensional signal under consideration, once its corresponding one-dimensional signals have been reconstructed. If the value of the signal on a $(2 N+1) \times(2 N$ +1 ) grid is needed, our proposed strategy is to determine its value at the intersections of sampling lines and $(2 \mathrm{~N}+$ 1) equally spaced horizontal or vertical lines, reconstruct the one-dimensional signals associated with these vertical or horizontal ones, and determine the signal value at $(2 \mathrm{~N}$ +1 ) equally spaced points on each horizontal or vertical line. Reconstruction of the one-dimensional signals associated with the horizontal or vertical lines is possible because each one intersects all the sampling lines at $(2 \mathrm{~N}$ +1 ) points. That is, if inequality (6) of Theorem 8 is satisfied, then each of the horizontal lines intersects the sampling lines at $(2 N+1)$ points, and if inequality $(7)$ is satisfied, then each of the vertical lines intersect the sampling lines at $(2 \mathrm{~N}$ $+1)$ points.

Let us now describe the iterative algorithm for reconstruction of one-dimensional signals associated with sampling lines. The algorithm is rather similar to that of Section IV-B. 1 in the sense that it iterates between space- and frequency-domain constraints by imposing appropriate constraints on the signal in each domain. If the locations of all the crossings of a one-dimensional continuous BLP signal $g(z)$ with $N_{t}$ thresholds $t_{1}<t_{2} \cdots<t_{N_{t}}$ are known, and the signal is known to be in the range $\left[t_{0}, t_{N_{t}+1}\right]$, then the intensity range of an arbitrary point $z_{0}$ can easily be deduced. This process is shown pictorially in Fig. 3(c). ${ }^{14}$ For a BLP signal with $2 N+1$ Fourier harmonics, the intensity range of $M>2 N+1$ equally spaced points of the signal constitute the space-domain constraint and the bandlimitedness of the signal constitutes the frequency-domain constraint. Similar to the two-dimensional iterative algorithm of the previous section, by iterating between the frequency and space domain, the algorithm can be shown to converge. In fact, Theorem 10 guarantees its convergence to a solution satisfying the constraints in both domains. As long as $M$ is finite, there might be many $M$-point real sequences satisfying both conditions. However, in the limit as $M \rightarrow \infty$, the solution set will contain exactly one sequence, as long as

\footnotetext{
${ }^{13}$ Examples of such reconstructions are included in [30].

${ }^{14}$ To derive the correct range information for any point on a line, we need a minimum of two samples corresponding to two different thresholds; samples corresponding to one threshold are incapable of resolving the sign ambiguity of the one-dimensional signal under
} consideration. 


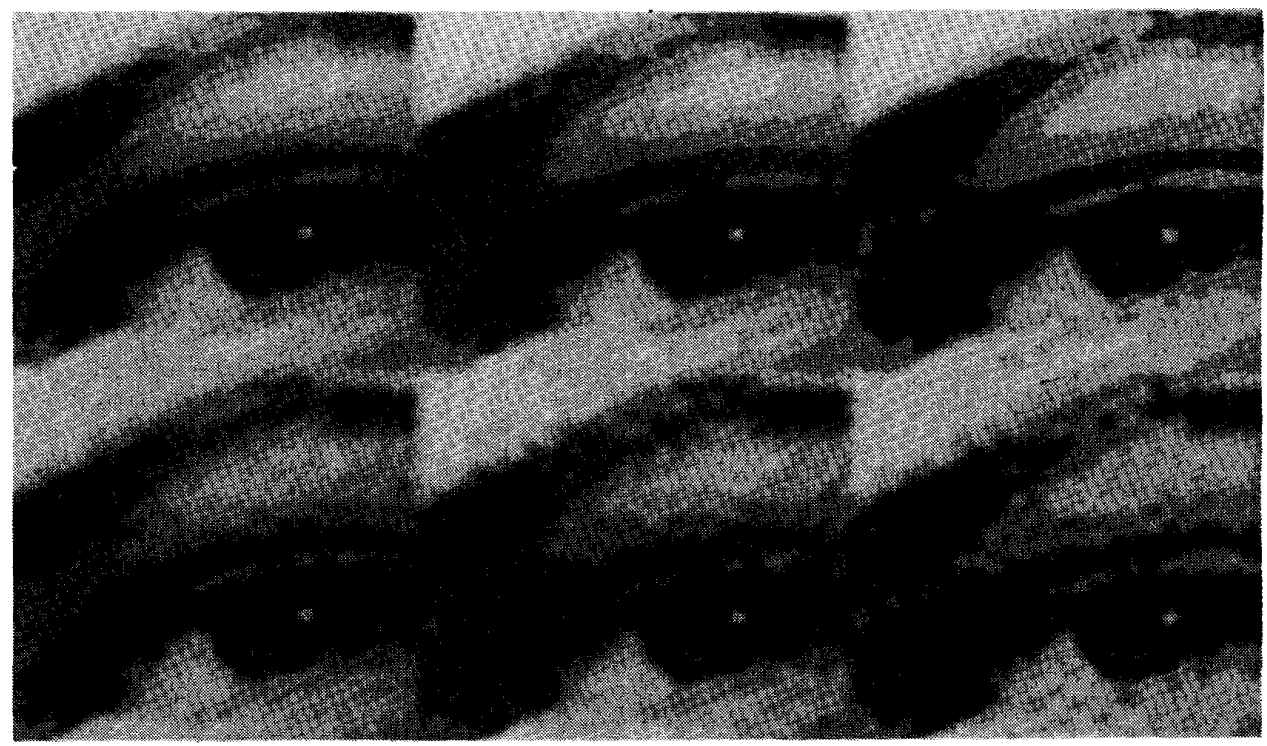

(a)

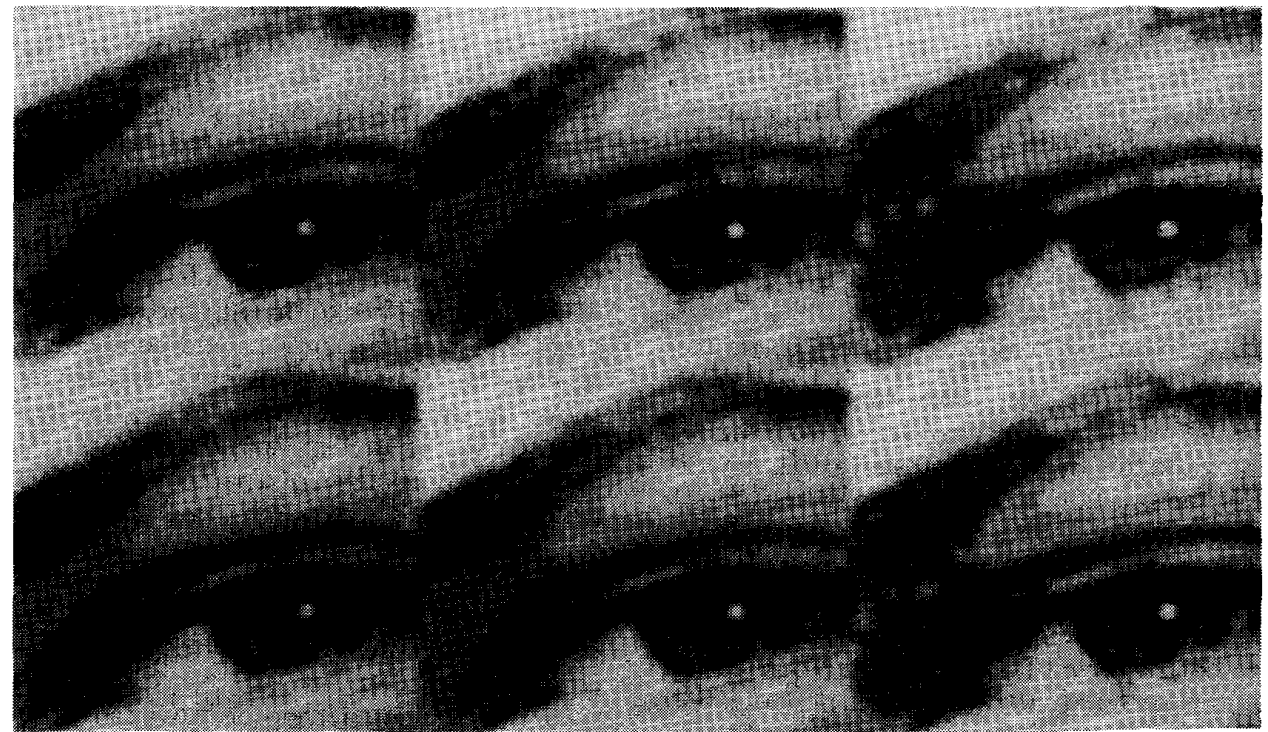

(b)

Fig. 4. (a) Reconstructed version of original eye picture from its implicit samples via iterative algorithm; Number of thresholds is 4, 6, and 8 , increasing from right to left; and number of equally spaced samples on lines $M$ is 31 (no reconstruction), 32, 64, 128, increasing from top to bottom. (b) Reconstructed version of eye pictu re from its semi-implicit samples via iterative algorithm; number of thresholds is $4,6,8$, increasing from right to left; number of equally spaced samples on lines increases from top to bottom and is 64 and 128 .

the number of samples on a given line exceeds the number of Fourier coefficients of the one-dimensional signal (because of the uniqueness of. Fourier series).

Fig. 4(b) shows the reconstructed version of the eye picture from the intersections of equally spaced lines of unit slope with 4,6 , and 8 theshold contours, via 20 iterations of the iterative algorithm for $M=64128$. In this case, since all the nodes of the $(2 N+1) \times(2 N+1)$ square grid of the two-dimensional signal are located on the sampling lines, interpolation from the one-dimensional signal to the original two-dimensional one is relatively straightforward. The relaxation parameters were chosen to be $\lambda_{1}=1, \lambda_{2}=1.75$. As seen, increasing the number of thresholds or $M$ improves the quality of reconstruction. Due to its lenient storage 


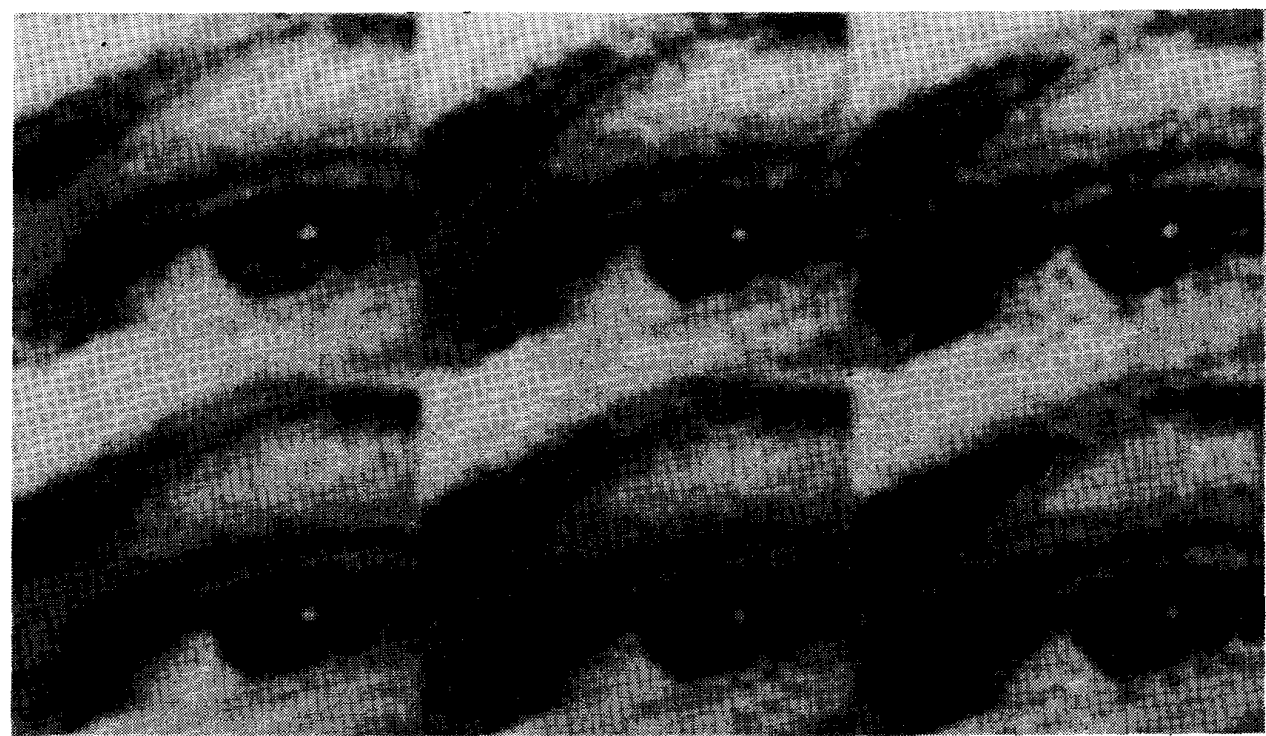

(c)

Fig. 4. (Continued.)

requirements, the iterative algorithm of this section can also be used to recover relatively large images. An example of such reconstruction is shown in Fig. 5(c), where the vegas picture is recovered from the intersections of 128 equally spaced lines of unit slope with eight threshold contours. The number of equally spaced samples on each line was 512 , and the relaxation parameters ${ }^{15}$ were $\lambda_{1}=1, \lambda_{2}=1.75$. In Section $V$, we discuss the quantization characteristics of the iterative algorithms.

\section{Preliminary Speculations on Quantization Properties}

In the last two sections, we have proposed various approaches to the problem of reconstruction from multiple-level crossings. In this section, we present a preliminary investigation of the position and amplitude quantization requirements of the sampling/reconstruction schemes as a function of the number of thresholds. A thorough investigation of these quantization characteristics involves extensive dealing with coding issues and experiments. Since we have not examined these issues rigorously, the discussions in this section tend to be rather preliminary, and most of the conclusions are somewhat tentative. However, these speculative results can be used as a starting point for further research in the areas of multidimensional signal representation and image coding. We begin with quantization properties of the linear least-squares approach in Section $V-A$, and continue with those of the iterative algorithms in Section V-B.

\section{A. Linear Least-Squares Approach}

This section includes a preliminary investigation of the way in which the number of thresholds affect position and amplitude quantization characteristics of reconstruction

\footnotetext{
${ }^{15}$ Some of the sampling lines in the reconstruction examples of this section did not contain as many threshold crossings as the number of Fourier harmonics of their corresponding $1 D$ signal.
}

via the linear least-squares approach. We have chosen the $\mathrm{QR}$ decomposition to solve the least-squares problem primarily because of its robustness. In Section V-A.1, we discuss the quantization characteristics of the semi-implicit and implicit sampling strategies, and in Section V-A.2, we show that, under certain circumstances, Nyquist sampling simply becomes a special case of the semi-implicit and implicit sampling strategies. Before approaching these, however, we need to address a few issues.

The first issue has to do with the fact that quantization procedures for the implicit and semi-implicit sampling schemes are somewhat different from each other. Fig. 6(a) shows quantization of samples of threshold contours obtained via the implicit sampling approach. As shown, the quantized coordinates of a sample associated with a particular threshold are chosen to be the coordinates of the center point of the square the sample falls in. For situations in which two or more samples corresponding to different thresholds fall into the same quantizing square, the sample closest to the center of the square is kept and the remaining ones are discarded.

For the semi-implicit approach, we can take advantage of the geometry of the sampling lines. As shown in Fig. 6(c), in a sampling scenario with $N_{/}$sampling lines, our strategy for specifying the location of a given sample has been to use $\log _{2} N_{l}$ bits to specify the line it falls on, and $b$ bits to specify its location along the sampling line. It is important to mention that there are many other ways to quantize the position of the samples for both the semi-implicit and implicit sampling schemes, and our proposed coding schemes are almost certainly not optimal.

The second issue that needs to be addressed is the way in which the number of reconstruction samples influences reconstruction robustness. Although the theoretical results of Section III provide sufficient conditions for unique recovery of BLP signals, using more interpolation points than the minimum required by these theoretical results improves the robustness of reconstruction and thus 


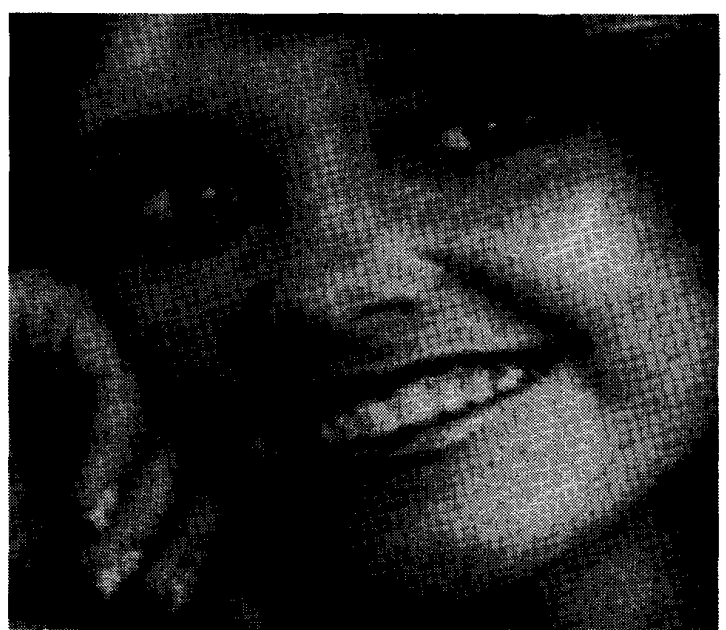

(a)

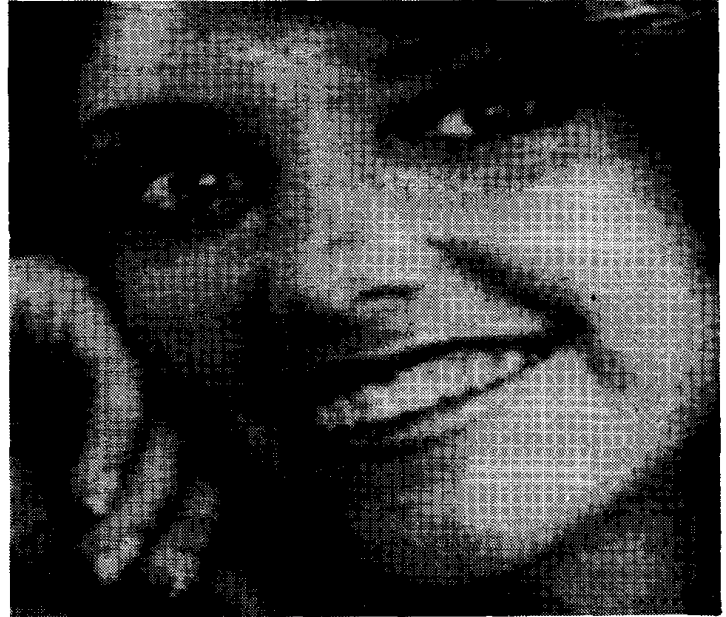

(b)

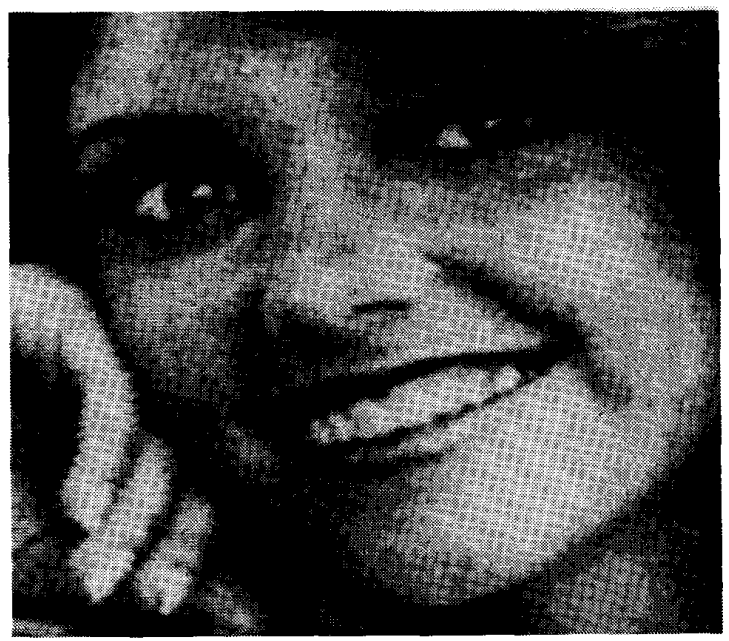

(c)

Fig. 5. (a) Original $256 \times 256$ vegas picture with $127 \times 127$ region of support in Fourier domain. (b) Reconstructed version of vegas picture from its implicit samples of eight level crossings via iterative algorithm. (c) Reconstructed version of vegas picture from its semiimplicit samples of eight level crossings via iterative algorithm.

decreases the accuracy needed for specifying the location of the crossings. We have found experimentally that, although for a fixed quality of reconstruction increasing the number of samples will initially decrease the number of position bits per sample, beyond a certain point it increases the total number of bits used to represent a signal [30]. In investigating the effects of the number of thresholds on quantization characteristics when the linear least-squares reconstruction is used, we have chosen to keep the number of reconstruction samples at a fixed level in all the experiments. Furthermore, to limit the computational intensity of the investigation, we have chosen to carry out all our experiments on only one picture with $15 \times 15$ region of support in the Fourier domain. This picture, which is referred to as "eye.Ip," is more or less the lowpass version of the original eye picture shown in Fig. 2(a). A more thorough study would obviously utilize a large number of images with different support regions.
1) Quantization Characteristics as a Function of the Number of Thresholds: Fig. 7(a) and 7(b) show mean square error (mse) versus the normalized number of amplitude and position bits as a function of the number of thresholds, for reconstruction of the eye.Ip picture via a linear least-squares method and QR decomposition. Fig. 7(a) corresponds to the semi-implicit sampling strategy with equidistant lines of unit slope, and Fig. 7(b) corresponds to the implicit sampling strategy. The mean square error between the original and reconstructed image is defined by:

$$
\text { mse }=\frac{1}{(2 N+1)^{2}} \sum_{k_{x}=-N}^{N} \sum_{k_{y}=-N}^{N}\left[F\left(k_{x}, k_{y}\right)-\tilde{F}\left(k_{x}, k_{y}\right)\right]^{2}
$$

where $F\left(k_{x}, k_{y}\right)$ and $\tilde{F}\left(k_{x}, k_{y}\right)$ correspond to the Fourier coefficients of the original and reconstructed images, respectively. We have found experimentally that the quality of the reconstructed image becomes almost indistinguishable from the original one when mse $\leq 0.1$. The normalized 


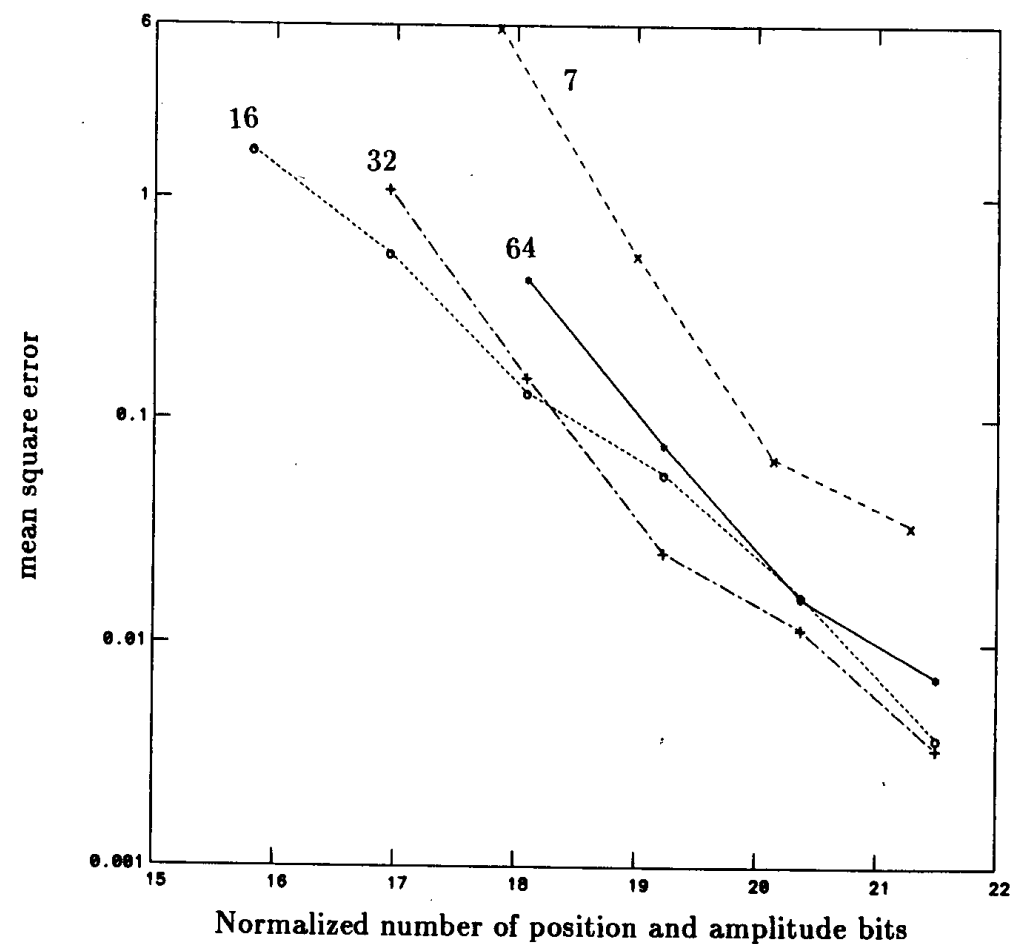

(a)

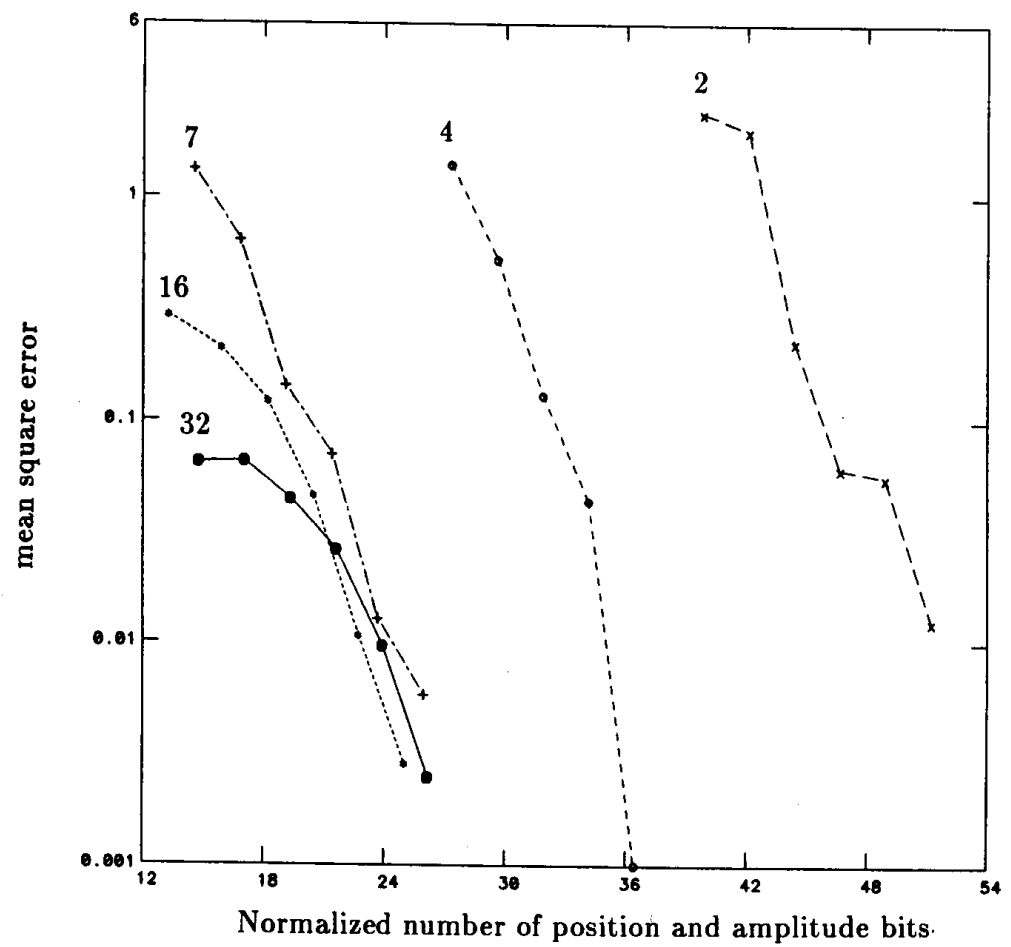

(b)

Fig. 6. (a) Quantization procedure for implicit samples of linear least-squares approach. (b) Position-quantized samples occupy all $N^{2}$ nodes of a $N \times N$ grid if number of thresholds are large enough. (c) Position-quantized samples obtained via semi-implicit sampling for linear least-squares reconstruction. (d) If we have $N$ equidistant horizontal or vertical sampling lines, and number of thresholds is large enough, position quantized samples will occupy all $N^{2}$ nodes of $N \times N$ grid. 


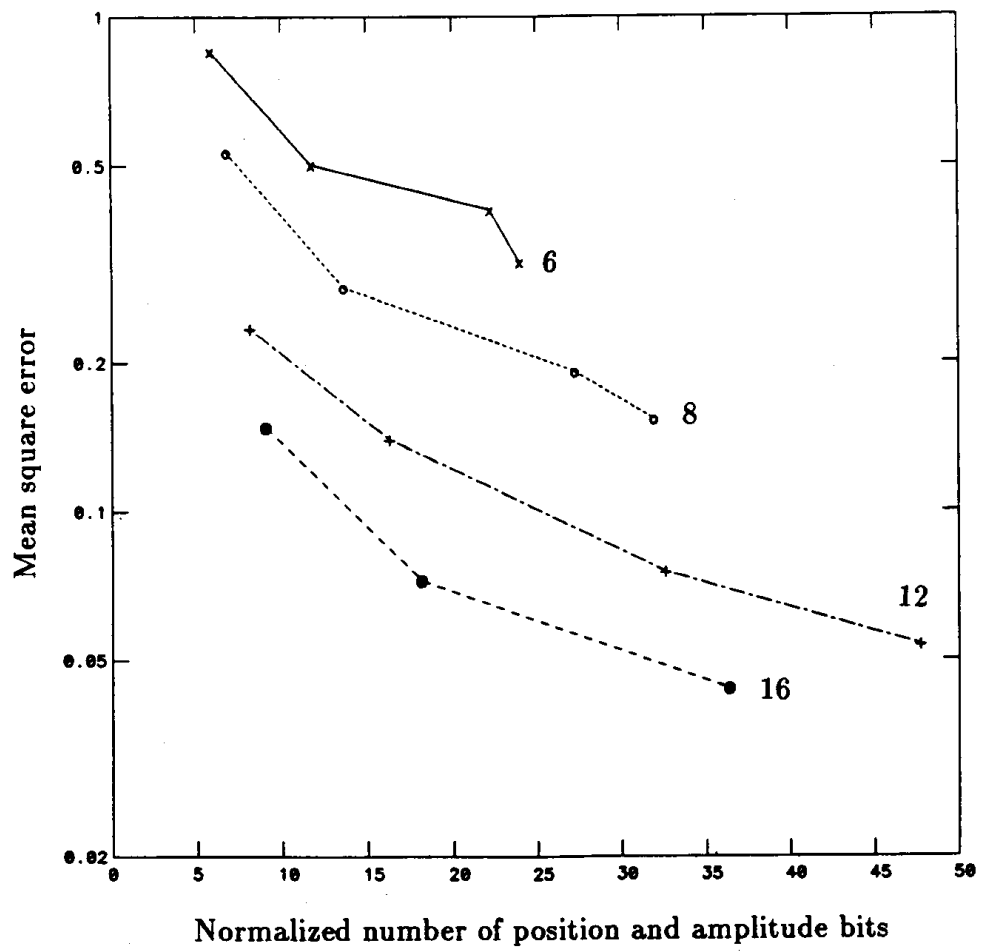

(c)

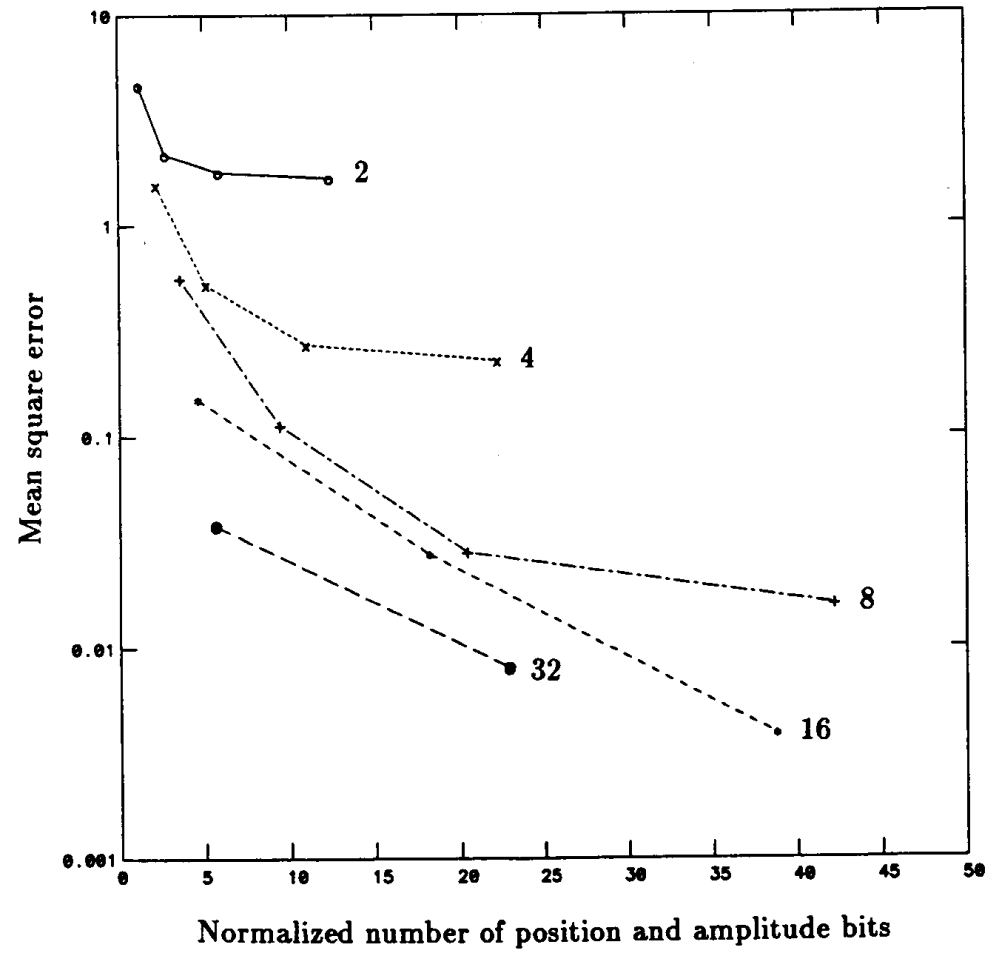

(d)

Fig. 6. (Continued.) 


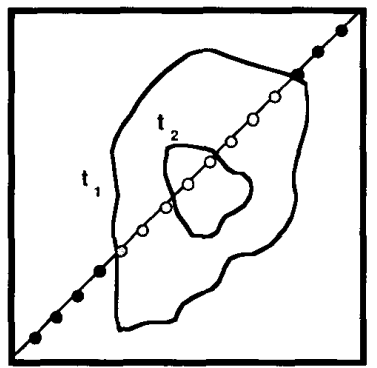

(a)

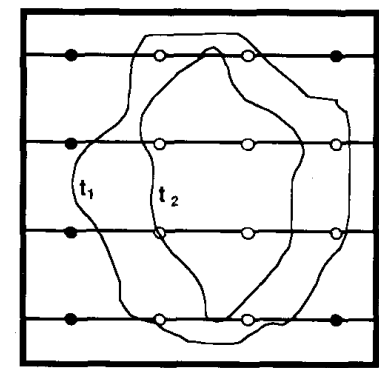

(b)
- points with intensity $<t_{1}$

0 points with intensity $>t$, and $<t_{2}$

$\circ$ points with intensity $>t_{2}$

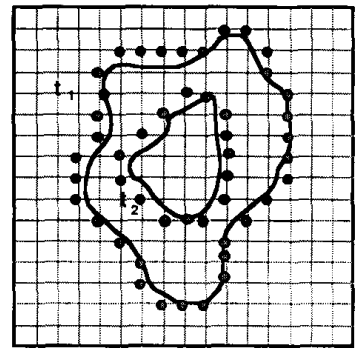

(c)

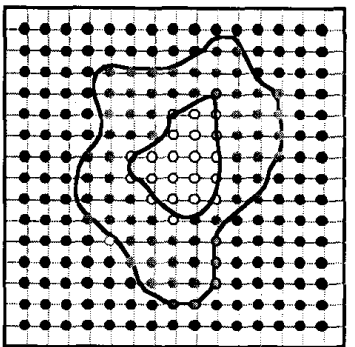

(d)

$$
t_{1}<t_{2}
$$

- points with intensity $<t$,

- points with intensity $>t$, and $<t_{2}$

- points with intensity $>t_{2}$

Fig. 7. Plot of mse versus normalized number of position and amplitude bits as function of number of thresholds for reconstruction of eye.Ip picture. (a) Via 256 semi-implicit samples and linear least-squares method. (b) Via 256 implicit samples and linear least-squares method. (c) Via semiimplicit samples of iterative algorithm. (d) Via implicit samples of iterative algorithm.

number of position and amplitude bits is defined as
The slopes of the curves shown in Fig. 7(a) and $7(\mathrm{~b})$ are negative, indicating that the quality of the reconstruction is improved as $B$ is increased. In addition, for fixed mean square error above 0.1 , the spacing between the curves apparently decreases from right to left, indicating that the improvement in the quality of reconstruction decreases as the number of thresholds gets larger. For instance, in curves of Fig. 7(a) (semi-implicit sampling), increasing the number of thresholds for a fixed mean-square error does not necessarily result in a decrease in the normalized number of position and amplitude bits. For curves of Fig. 7(b) (implicit sampling), the "optimum" number of thresholds which results in minimum total (position and amplitude) number of bits varies as a function of mse. For instance, for $0.2 \leq$ mse $\leq 1$, it is 7 ; for $0.06 \leq \mathrm{mse} \leq 0.2$, it is 16 ; and for 0.02 $\leq \mathrm{mse} \leq 0.06$, it is 32 .

2) Relationship of Semi-Implicit and Implicit Sampling to Nyquist Sampling: In explicit sampling a function is represented by its samples at prespecified points. An important example of such a technique is Nyquist sampling, which involves amplitude specification of the signal at equally spaced points. In this section, we will show that, under certain circumstances, the locations of samples used for reconstruction via the linear least-sqaures approach become identical to those of Nyquist sampling. We will begin with the semi-implicit sampling strategy.

Semi-implicit sampling: As discussed in Section III-A, reconstruction of an $N \times N$ signal from its crossings with $N_{t}$ arbitrary functions, via the semi-implicit sampling strategy, consists of sampling the function crossings of the signal along lines of rational slope, and quantizing the position of the samples to $b$ bits along the line. The above process is shown pictorially in Fig. 6(c). As shown in Fig. 6(d), if the crossing functions are chosen to be constants (i.e., the reconstruction samples are chosen from level crossings of the signal), the sampling lines are chosen to be equally spaced horizontal or vertical lines, and $2^{b}=N$, then the locations of the $N^{2}$ position quantized samples correspond to the nodes of a $N \times N$ grid or, equivalently, the location of Nyquist samples. Note that for the preceding conditions to hold, the number of thresholds used for reconstruction must be large enough so that each of the $N$ horizontal (or vertical) lines contain $N$ samples after quantizing the $x$ (or v) position of the samples to $\log _{2} N$ bits.

$$
B=\frac{\text { number of position and amplitude bits per sample } \times \text { number of samples }}{\text { number of Fourier coefficients }} .
$$

The number of amplitude bits per sample is defined to be $\log _{2}$ (number of thresholds). For all the curves of Fig. 7(a) and $7(b)$, the number of reconstruction samples was 256 . Moreover, since all the curves were obtained from a single image, namely eye.lp, the number of Fourier coefficients is identical in each case. Thus, in effect, these curves represent the mean square error as a function of the number of position and amplitude bits per sample for different choices of the number of thresholds. As we will see later, unlike the linear least-squares approach, increasing the number of position bits per sample for the iterative automatically increases the number of samples used for reconstruction. This is the reason behind choosing the normalized number of position and amplitude bits as a "metric" for comparing various algorithms and sampling strategies.
Although locations of Nyquist and semi-implicit samples become identical under these characteristics, it is important to bear in mind that these sampling sets are inherently different from each other. In Nyquist sampling, the amplitude information is quantized at the nodes of a $N \times N$ grid, whereas in semi-implicit sampling the nodes of this $N \times N$ grid correspond to position-quantized samples of level crossings. In other words, Nyquist sampling corresponds to amplitude quantization at prespecified points, and reconstruction from level crossings via semi-implicit sampling corresponds to position quantization along sampling lines at prespecified amplitudes.

Implicit sampling: As discussed in Section III-B, reconstruction of a $N \times N$ signal from implicit samples of its $N_{t}$ level crossings via the linear least-squares approach con- 
sists of quantizing the threshold contours by superimposing a $2^{b} \times 2^{b}$ grid over the signal in the space domain, and choosing $N_{s}>N^{2}$ of the $1 / 2^{b} \times 1 / 2^{b}$ squares whose centers correspond to quantized reconstruction samples. This process is shown pictorially in Fig. 6(a). As seen in Fig. 6(b), for a sufficiently large number of thresholds, all the $2^{2 b}$ squares associated with the $2^{b} \times 2^{b}$ grid will have a threshold value associated with them. Moreover, if the grid size is the same as the signal size, i.e., $2^{b}=N$, the number of reconstruction samples, $M$, becomes equal to $N^{2}$, and the locations of position-quantized samples of level crossings correspond to nodes of a $N \times N$ grid or, equivalently, the locations of Nyquist samples. Thus Nyquist sampling corresponds to amplitude quantization at prespecified points, and reconstruction from level crossings corresponds to position quantization at prespecified amplitudes.

\section{B. Iterative Approach}

The quantization properties of the iterative algorithms are somewhat different from those of the linear leastsquares approach. In Section V-B.1, we present a preliminary investigation of amplitude and position quantization requirements of the semi-implicit and the implicit sampling strategies. Section V-B.2 shows that, under certain circumstances, Nyquist samples become identical to semi-implicit and implicit samples used for reconstruction via the iterative algorithms.

1) Quantization Characterization as a Function of the Number of Thresholds: We begin with characterization of the semi-implicit approach.

Semi-implicit sampling: In Fig. 7(c) we show the mean square error between the origional eye.lp picture and its reconstructed version via the iterative algorithm as a function of the normalized number of position and amplitude bits. As indicated, the number of thresholds associated with the four curves is $6,8,12$, and 16 . For each curve, the thresholds were chosen with equal spacing between 0 and 256 . The points on each curve correspond to different numbers of equally spaced points on the sampling lines, i.e., $M=32$, 64,128 . In addition, the sampling lines were chosen to be equidistant and of unit slope. ${ }^{16}$ The $y$ axis corresponds to mse, as defined by (20), and the $x$ axis indicates normalized number of position and amplitude bits used for the particular reconstruction at hand.

Because of the inherent structure of the samples used by the iterative algorithm, there are a variety of ways to represent the signal under consideration and to arrive at the number of quantization bits. The most straightforward way is to quantize the locations of threshold crossings on the sampling lines to $\log _{2} M$ bits in such a way that the space domain constraint for the $M$ equally spaced points on each of the sampling lines can be derived easily. Using this strategy, the total number of amplitude and position bits for representing the signal is $\left(\log _{2} M+\log _{2} N_{t}+\log _{2} N_{l}\right)$ $\sum_{i=1}^{N_{t}} N_{i}$, where $N_{i}$ denotes the number of intersections of the $i$ th threshold crossings with all the sampling lines, $N_{t}$

${ }^{16} \mathrm{Although}$ the theoretical results of Section III-A require only 15 sampling lines of unity slope, to be able to use the FFT with a power of 2 for the interpolation part of the iterative algorithm (i.e., interpolation from recovered one-dimensional signals to a square grid on the two-dimensional one) we chose 16 equally spaced sampling lines. denotes the number of thresholds, $N_{1}$ denotes the number of sampling lines, and $M$ denotes the number of equally spaced points on each of the sampling lines.

An alternative strategy for representing the signal is to specify the intensity range for each of the equally spaced points on the sampling lines. More specifically, if the number of thresholds is $N_{t}$, then the value of the signal at any given point lies in one of the $\left(N_{t}+1\right)$ intervals corresponding to the $N_{t}$ thresholds. In this case, the total number of bits used to represent the signal is given by $M N_{1} \log _{2}\left(N_{t}+\right.$ 1). Clearly, this second quantization strategy outperforms the first one for large values of $N_{t}$ and small values of $M$. Our strategy in determining the total number of position bits for the abscissa of Fig. 7(c) has been to choose the minimum of the preceding two quantization strategies. We have found experimentally that we would reach similar conclusions regardless of the quantization strategy used.

Having discussed the details of generating the curves shown in Fig. 7(c), it is now appropriate to make a few observations and comments regarding their shapes. As we would expect, the slope of each curve is negative, indicating that for fixed number of thresholds the mean square error decreases as $M$ is increased. In addition, for fixed $M$, the mean square error is decreased as the number of thresholds is increased. An interesting question to address is whether or not there is an "optimum' number of thresholds for which the lowest number of amplitude and position quantization bits is achieved. As Fig. 7(c) shows, this "optimum" number varies as a function of the mean square error. For instance, for mse in the range $[0.53,0.85]$, it is $6-8$, and for mse in the range $[0.15,0.23]$, it is $12-16$. Thus the "optimum" number of thresholds is a decreasing function of mse. In the next section, we will investigate iterative reconstruction using implicit sampling.

Implicit sampling: In this section we investigate quantization properties of the iterative reconstruction algorithm for the implicit sampling strategy. Similar to the iterative reconstruction algorithm of the semi-implicit sampling, there are several strategies one might take to arrive at the total of amplitude and position quantization bits for representing a given image via the implicit sampling scheme. Since in each of these strategies we are encoding the quantized contours corresponding to different thresholds, almost all of the efficient coding schemes for two-tone images proposed and studied by many researchers [38], [39] can be used for representing our images. The most obvious way of encoding the boundary points of a threshold contour quantized to $b$ bits is to use $2 b$ bits for the $x$ and $y$ coordinates of each point. Using this strategy, the total number of bits required to specify $N_{t}$ threshold contours is $\left(\log _{2} N_{t}\right.$ $+2 b)\left(\sum_{i=1}^{N_{t}} N_{i}(b)\right)$, where $N_{i}(b)$ denotes the number of quantized boundary points on a $2^{b} \times 2^{b}$ quantization grid for the $i$ th threshold. A second and more efficient way of representing the boundary points is to follow the boundary, i.e., to do contour tracing. Since the image is quantized on a $2^{b}$ $\times 2^{b}$ square grid, each pel has only eight neighbors. Therefore it is sufficient to use 3 bits to indicate where the next boundary point is. Of course, to get on each boundary, we need to specify the position of an initial point. Thus, ignoring the additional bits required to get to the initial points on the boundaries and to specify their associated threshold value, total number of bits required for specifying an image is $3 \sum_{i=1}^{N_{t}} N_{i}(b)$. 
Our third encoding strategy involves specifying the range of the signal for each node of the $2^{b} \times 2^{b}$ quantization grid. This strategy is suitable for sampling schemes with large number of thresholds and coarse quantization of the contours. The total number of bits required for specifying an image via this encoding technique is $2^{2 b} \log _{2}(N+1)$.

As far as efficiency of these encoding strategies goes, clearly the second one outperforms the first one, and the relative efficiencies of the second and third ones depend on the form of $N_{i}(b)$. Intuitively, we would expect $N_{i}(b)$ to be proportional to $2^{b}$, since the number of boundary points of quantized threshold contours is doubled as the size of quantizing grid is increased from $2^{b} \times 2^{b}$ to $2^{b+1} \times 2^{b+1}$. Thus, for small values of $b$ and large values of $N_{t}$, the third strategy outperforms the second one. As it turns out, our major conclusions are more or less independent of the actual encoding scheme used; so our adopted strategy has been to choose the minimum of second and third ones to represent images.

Having described the quantization strategy, we will now examine how the number of thresholds affect the required number of position bits and the quality of reconstruction. Fig. 7(d) shows a plot of the mean square error versus the normalized number of position and amplitude bits as a function of the number of thresholds. The five curves of Fig. 7 (d) correspond reconstruction from different numbers of thresholds. Various points on each curve correspond to reconstruction with different values of grid size. The slopes of the curves are negative, indicating that the quality of reconstruction improves as the quantization grid becomes finer. In addition, the number of thresholds which results in smallest number of quantization bits is a function of mse. For instance, if we are interested in reconstructing signals with mse $\leq 0.556$, then the optimal number of thresholds is between 8 and 16. Finally, comparing Fig. $7(\mathrm{c})$ and $7(\mathrm{~d})$ it seems that, for fixed quality of reconstruction via iterative algorithms, implicit sampling results in lower number of bits than semi-implicit sampling with lines of unit slope.

2) Relationship to Nyquist Sampling: In this section, we will show that, under certain circumstances, the samples used for reconstruction via the iterative algorithms become identical to those of Nyquist sampling. We begin with the semi-implicit sampling strategy.

Semi-implicit sampling: As shown in Fig. 3(c), the iterative algorithm for the semi-implicit sampling strategy utilizes position-quantized semi-implicit samples to derive the space domain constraint. Therefore, the input to the iterative algorithm can be represented by the intensity range of equally spaced points on the sampling lines. Since all the intersections of sampling lines with the threshold contours are utilized, the intensities of the $M$ equally spaced points lie in one of $N_{t}+1$ intervals defined by the $N_{t}$ thresholds. In addition, suppose that we have $N$ equally spaced horizontal or vertical sampling lines for a signal with $N \times N$ support region in the Fourier domain. As Fig. 3(d) shows, if $M$ $=N$, then the $N$ equally spaced samples on $N$ horizontal or vertical lines correspond to nodes of a $N \times N$ grid, and since the amplitude of each node is in one of the $\left(N_{t}+1\right)$ intervals corresponding to $N_{t}$ thresholds, the sampling set becomes identical to $\log _{2}\left(N_{t}+1\right)$ bit amplitude-quantized Nyquist samples.

Implicit sampling: Recall from Section V-B.1 that the quantization procedure for iterative reconstruction of a $N$ $\times N$ signal from $N_{t}$ level crossings via the implicit sampling strategy consists of deriving the amplitude range for the nodes of a $2^{b} \times 2^{b}$ grid by utilizing the quantized threshold contours. Pictorially, this is shown in Fig. 3(a). Since the iterative algorithm needs all the quantized contours associated with all the thresholds to derive the correct space domain constraint, the intensity of each node of the grid lies in one of the $N_{t}+1$ intervals defined by the $N_{t}$ thresholds. Thus, as shown in Fig. 3(b), we can think of the nodes of the grid being amplitude-quantized to $\log _{2}\left(N_{t}+1\right)$ bits. In addition, if the size of the grid, $2^{b}$, assumes its minimum possible value, i.e., $N$, then the sampling set becomes identical to $\log _{2}\left(N_{t}+1\right)$ bit amplitude-quantized Nyquist samples.

\section{Conclusion}

Our main goal in this paper has been to discuss sampling strategies for reconstruction of multidimensional signals from multiple level threshold crossings, whose characteristics lie in between Nyquist sampling and the zero-crossing representation proposed by Curtis and Oppenheim [1], [17]. Our approach has been to formulate the problem in terms of multivariate interpolation theory. Our main theoretical results in this paper deal with two major sampling strategies.

Our first strategy, described in Section III-A, consisted of imposing restrictions on the location of interpolation points used for recovery of the bivariate polynomial associated with the signal under consideration. To this end, we developed theoretical results on multivariate polynomial interpolation theory using algebraic geometric concepts. We then used these results to derive the semi-implicit sampling strategy to provide sufficient conditions under which multidimensional BLP signals can be recovered from their nonuniform samples on lines of rational slope. To utilize these results in the context of reconstruction from level crossings, the nonuniform samples were chosen at the intersection of sampling lines with level crossings. As shown in [30], the semi-implicit sampling strategy can also be applied to a variety of other problems, such as reconstruction from crossings with arbitrary functions and reconstruction from projections.

The major drawback of the line-sampling strategy for reconstruction from level crossings is the fact that, for a small number of thresholds, we are not guaranteed to get enough intersections between the sampling lines and level crossings to satisfy the conditions of Theorem (8). To overcome this difficulty, in Section III-B, we proposed the implicit sampling strategy for reconstruction from an arbjtrarily small number of thresholds. The major result in Section III-B states that for almost all signals with $N \times N$ region of support in the Fourier domain, almost all $k>0$ points from its $\gamma$ level crossings and $N^{2}-k$ points from its $\delta$ level crossings are sufficient to uniquely specify it. This result was extended to situations where the number of thresholds is larger than 2, and to the problem of reconstruction from crossings with functions whose bandwidths lie within the bandwidth of the signal.

Having developed the semi-implicit and implicit sampling strategies, we then proposed a number of reconstruction algorithms in Section IV. The most straightforward way of carrying out reconstructions for both the semi-implicit and implicit sampling strategies is to solve a linear system 
of equations to find the Fourier-series coefficients associated with the signal. Although the linear least-squares approach can result in stable reconstructions, its storage requirements are rather demanding. To overcome this problem, we proposed the iterative algorithms for both semi-implicit and implicit sampling strategies.

In Section V, we presented a preliminary investigation of the quantization properties of the reconstruction algorithms as a function of the number of thresholds. In Section $V-A$, we found that for a fixed quality of reconstruction and fixed number of reconstruction samples, the quantization characteristics of the linear least-squares approach initially improve as the number of thresholds is increased. However, for large number of thresholds, further increase in the number of thresholds does not necessarily lead to fewer quantization bits. As we saw in Section V-B, the quantization properties of the iterative algorithms suggest that the "optimum" number of thresholds which results in the smallest number of position and amplitude bits is highly dependent on the quality of the reconstructed images. More specifically, as Figs. 7(c) and 7(d) show, for smaller values of the mean square error, the "optimum" number of thresholds is larger. It is important to mention that the results presented in Section $V$ are extremely preliminary and that the conclusions are tentative. Our hope is that these speculative results can be used as a starting point for further research in the applications of the theory to areas of multidimensional signal representation and image coding.

Our goal in this paper has been to derive sampling schemes whose bandwidth and dynamic range characteristics lie in between those of Nyquist and zero-crossings sampling. While recovery of an $N \times N$ signal from its $N y-$ quist samples requires minimum $\left(\log _{2} N\right)$ number of position bits and large number of amplitude bits, and recovery from zero crossings requires large number of position bits and minimum (1) number of amplitude bits, the position and amplitude quantization requirements of our sampling strategies for reconstruction from multiple level crossings lie in between these two extremes. In fact, the experimental results of Section $V$ seem to suggest that the optimal number of thresholds, which results in minimum number of total amplitude and position bits, is neither infinite, as it is with Nyquist sampling, nor is it one, as is the case with zerocrossing sampling. Indeed, this optimum number depends on a variety of factors, such as

- quality of reconstruction,

- the specific sampling strategy used, i.e., semi-implicit or implicit sampling,

- the specific reconstruction strategy,

- the number of reconstruction samples.

Finally, as we saw in Sections V-A.2 and V-B.2, representation of two-dimensional signals via their amplitude-quantized explicit Nyquist samples is intimately related to their position-quantized implicit or semi-implicit samples. The results of Section $V$ seem to indicate that not only does the amplitude and position quantization characteristics of our sampling and reconstruction schemes lie in between those of Nyquist and zero-crossings, but also, under certain circumstances, semi-implicit and implicit sampling strategies become a special case of Nyquist sampling. In short, reconstruction from multiple-level threshold crossings has bridged the gap between explicit, semi-implicit, and implicit sampling strategies, has unified seemingly unrelated sampling schemes, and has provided us with a spectrum of sampling techniques for multidimensional signals.

\section{APPENDIX A}

Formal Statements of Some of the Theorems

This appendix contains the formal statements of the theorems cited in the body of the paper. Theorems which represent new contributions of this paper are indicated with an asterisk. Proofs are included for these theorems only and are contained in Appendices $B$ and $D$ with some associated discussion.

Logan's theorem provides conditions for unique recovery of one-dimensional signals from their zero crossings.

Theorem 1 (Logan [5]): Consider two real-valued bandpass functions $h_{1}(t)$ and $h_{2}(t)$, having spectra confined to $\left[\omega^{\min }, \omega^{\max }\right]$ and $\left.\left[-\omega^{\max },-\omega^{\min }\right]\right)$, where $0<\omega^{\min }<\omega^{\max }<$ $2 \omega^{\text {min }}$. If they have no free zeros other than real simple free zeros, then

$$
\operatorname{sgn}\left[h_{1}(t)\right] \equiv \operatorname{sgn}\left[h_{2}(t)\right] \quad-\infty<t<\infty
$$

implies

$$
h_{1}(t) \equiv A h_{2}(t) .
$$

The free zeros of a real-valued bandpass function $h(t)$ are simply the common zeros of $h$ with its Hilbert transform.

The following theorem is an extension of Logan's result to two-dimensional signals which are bandpass in both directions.

Theorem 2 (Rotem and Zeevi [16]): Let $f(x, y)$ be a real square-integrable bandpass function in both dimensions whose Fourier transform $F\left(\omega_{x}, \omega_{y}\right)$ is zero for

$$
\omega_{x}^{\min }<\left|\omega_{x}\right|<\omega_{x}^{\max }, \quad \omega_{y}^{\min }<\left|\omega_{y}\right|<\omega_{y}^{\max }
$$

where

$$
0<\omega_{x}^{\min }<\omega_{x}^{\max }<2 \omega_{x}^{\min } . \quad 0<\omega_{y}^{\min }<\omega_{y}^{\max }<2 \omega_{y}^{\min } .
$$

Assume the following conditions are satisfied:

1) There exists a $y_{0}$ such that the one-dimensional function of $x, f\left(x, y_{0}\right)$ and its Hilbert transform have only real zeros of degree one in common.

2) There exists a sampling set $\left\{x_{k}\right\}$ for $B\left(\omega_{x}^{\min }, \omega_{x}^{\max }\right)$ with density greater than $\omega_{x}^{\max }-\omega_{x}^{\min } / \pi$ such that the onedimensional functions in $y$ given by $f\left(x_{k}, y\right)$ have only real zeros of degree one with their Hilbert transforms. $B\left(\omega_{x}^{\min }, \omega_{x}^{\max }\right)$ denotes the one-dimensional bandpass signals $g(z)$ whose fourier transform, $G(\omega)$, is zero for $|\omega|>\omega_{x}^{\max }$ and $|\omega|<\omega_{x}^{\min }$, and sampling set with density greater than $\omega_{x}^{\max }-\omega_{x}^{\min } / \pi$ implies

$$
\lim _{l \rightarrow \infty} \sup \frac{N(L)}{L}>\frac{\omega_{x}^{\max }-\omega_{x}^{\min }}{\pi}
$$

where $N(L)$ denotes the number of samples in the interval $(0, L)$. Then $f(x, y)$ is uniquely specified from its zero crossings to within a scale factor.

The following theorem is an extension of Logan's result to two-dimensional signals which are bandpass in one direction and lowpass in the other. 
Theorem 3 (Rotem and Zeevi [16]): Let $f(x, y)$ be a real square-integrable function which is bandpass in one direction and lowpass in the other. In other words, its Fourier transform $F\left(\omega_{x}, \omega_{y}\right)$ is zero for

$$
\omega_{x}^{\min }<\left|\omega_{x}\right|<\omega_{x}^{\max }, \quad\left|\omega_{y}\right|>\omega_{y}^{\max }
$$

where

$$
0<\omega_{x}^{\min }<\omega_{x}^{\max }<2 \omega_{x}^{\min } \quad 0<\omega_{y}^{\max }<\infty .
$$

If the following conditions are satisfied:

1) there exists a sampling set $\left\{y_{k}\right\}$ for one-dimensiona lowpass signals with bandwidth $\omega_{y}^{\max }$ such that the one-dimensional functions in $x, f\left(x, y_{k}\right)$ and their Hilbert transforms have only real zeros of degree one in common;

2) there exists a straight line $S$ of angle $\theta$ to the $x$ axis, where $\tan (\theta)<2 \omega_{x}^{\min }-\omega_{x}^{\max } / 3 \omega_{y}^{\max }$ such that the one dimensional signal along $S, f(s)$ has only real zeros of degree one in common with its Hilbert transform and satisfies

$$
\int_{-\infty}^{\infty} f(s) d s<\infty
$$

then $f(x, y)$ is uniquely specified up to a scale factor from its zero crossings.

As shown in the following, Benzout's theorem provides an upper bound for maximum common zeros of two bivariate polynomials.

Theorem 4 (Bezout [40], [47]): If two bivariate polynomials of total degree $R$ and $S$ given by

$$
\begin{aligned}
& p(x, y)=\sum_{i=0}^{R} \sum_{j=0}^{R-i} a(i, j) x^{i} y^{i} \\
& q(x, y)=\sum_{i=0}^{S} \sum_{j=0}^{S-i} b(i, j) x^{i} y^{i}
\end{aligned}
$$

have no common factors of degree greater than zero, then they have at most $R S$ common zeros.

The following theorem deals with unique specification of BLP signals from their zero crossings.

Theorem 5 (Curtis and Oppenheim [1]): Consider a real two-dimensional doubly periodic bandlimited signal

$$
f(x, y)=\sum_{k_{x}=-N}^{N} \sum_{k_{y}=-N}^{N} F\left(k_{x}, k_{y}\right) \mathrm{e}^{i 2 \pi\left(k_{x} x+k_{y} y\right)} .
$$

The polynomial representation of this signal is given by $g(w, z)=f(x, y) w^{N} z^{N}=\sum_{k_{x}=0}^{2 N} \sum_{k_{y}=0}^{2 N} F\left(k_{x}-N, k_{y}-N\right) w^{k_{x}} z^{k_{y}}$

where

$$
w=\mathrm{e}^{j 2 \pi x}, \quad z=\mathrm{e}^{i 2 \pi y} .
$$

If $g(w, z)$ is irreducible (nonfactorable), then $f(x, y)$ can be uniquely specified to within a scale factor from $16 N^{2}+1$ or more of its samples.

The following theorem is the modified version of Bezout's theorem. It provides an upperbound on the maximum number of finite common zeros of polynomials whose coefficients have rectangular region of support.
Theorem 6 (Zakhor and Izraelivitz [18]): Consider two bivariate polynomials $p(x, y) \in \Pi\left(N_{\left.x, N_{y}\right)}\right.$ and $q(x, y) \in$ $\Pi\left(M_{\left.x, M_{y}\right)}\right.$ of the form

$$
\begin{aligned}
& p(x, y)=\sum_{i=0}^{N_{x}} \sum_{j=0}^{N_{y}} a(i, j) x^{i} y^{j} \\
& q(x, y)=\sum_{i=0}^{M_{x}} \sum_{j=0}^{M_{y}} b(i, j) x^{i} y^{j} .
\end{aligned}
$$

If $p$ and $q$ have no common factors of degree greater than zero, then they have at most $N_{x} M_{y}+M_{x} N_{y}$ common zeros.

The following theorem deals with interpolation of polynomials whose coefficients have triangular region of support.

Theorem 7(Gasca and Maetzu [27]): Consider the distinct lines $I_{0}, \cdots, I_{R}$ with the set of distinct points

$$
\left\{\left(w_{i}^{(i)}, z_{j}^{(i)}\right) \mid j=0,1, \cdots, i\right\}
$$

on $I_{i}$. If none of the interpolation points $\left(w_{j}^{(i)}, z_{j}^{(i)}\right)$ are on the intersection of any two lines from $I_{0}, \cdots, I_{R}$, then for any data set

$$
\left\{t_{j}^{(i)} \mid j=0, \cdots, i ; \quad i=0,1, \cdots, R\right\}
$$

there is a unique bivariate polynomial $p \in \Pi_{R}$ such that

$$
p\left(w_{j}^{(i)}, z_{i}^{(i)}\right)=t_{j}^{(i)} \quad 0 \leq j \leq i, \quad 0 \leq i \leq R .
$$

The following theorem is the basis for the semi-implicit sampling strategy and provides conditions under which bivariate polynomials can be recovered from their samples on irreducible curves.

Theorem $8{ }^{*}$ (Section III-A.2): Let $\mathrm{c}_{0}, \mathrm{C}_{1}, \cdots, \mathrm{C}_{N_{\mathrm{c}}}$ be distinct bivariate irreducible polynomials with the maximum degrees of $C_{i}$ in $w$ and $z$ given by $M_{w}^{(i)}$ and $M_{z}^{(i)}$ and $N_{c}$ being an integer satisfying either of the following two conditions:

$$
\begin{gathered}
N_{\mathrm{w}}<\sum_{i=0}^{N_{\mathrm{c}}} M_{\mathrm{w}}^{(i)} \\
N_{\mathrm{z}}<\sum_{i=0}^{N_{\mathrm{c}}} M_{z}^{(i)}
\end{gathered}
$$

Define $A_{i}$ to be the set of

$$
S(i) \equiv M_{z}^{(i)}\left(N_{w}-\sum_{k=0}^{i-1} M_{w}^{(k)}\right)+M_{w}^{(i)}\left(N_{z}-\sum_{k=0}^{i-1} M_{z}^{(k)}\right)+1
$$

points on $C_{i}$ given by

$$
A_{i}=\left\{\left(w_{i}^{(i)}, z_{j}^{(i)}\right) \mid C_{i}\left(w_{j}^{(i)}, z_{j}^{(i)}\right)=0,0 \leq j<S(i)\right\} .
$$

If none of the interpolation points given by (27) are on the intersections of two or more of the $C_{i}^{\prime}$ s, then for any data set

$$
\left\{t_{i}^{(i)} \mid 0 \leq i \leq N_{\mathrm{c}}, 0 \leq j<S(i)\right\}
$$

there is no more than one bivariate polynomial of the form

$$
p(w, z)=\sum_{i=0}^{N_{w}} \sum_{j=0}^{N_{z}} a(i, j) w^{i} z^{j}
$$

such that

$$
\left.p\left(w_{i}^{(i)}, z_{j}^{(i)}\right)=t_{j}^{(i)} \quad 0 \leq i \leq N_{c}, \quad 0 \leq j<S(i)\right\} .
$$

The following theorem is the basis of the implicit sampling strategy. The definition of the polynomials and sets used in this theorem are given in section III-B. 
Theorem $9 *$ (Section $I I I-B)$ : The set of real zeros of the polynomial $\tilde{d}\left(w_{1}, \cdots, v_{(2 N+1)^{2}}\right)$ is of measure zero in the set $B(R)$ provided the following conditions hold.

1) $\tilde{A}_{\gamma}(\mathrm{R})$ and $\bar{A}_{\delta}(\mathrm{R})$ have maximal topological dimensions. ${ }^{17}$

2) The polynomials

$$
\begin{aligned}
& g_{\gamma}\left(W_{1}, W_{2}\right)=\sum_{k_{x}=0}^{2 N} \sum_{k_{y}=0}^{2 N} F_{y}\left(k_{x}-N, k_{y}-N\right) W_{1}^{k_{x}} W_{2}^{k_{y}} \\
& g_{\delta}\left(W_{1}, W_{2}\right)=\sum_{k_{x}=0}^{2 N} \sum_{k_{y}=0}^{2 N} F_{\delta}\left(k_{x}-N, k_{y}-N\right) W_{1}^{k_{x}} W_{2}^{k_{y}}
\end{aligned}
$$

with

$$
\begin{aligned}
& F_{\gamma}\left(k_{x}, k_{y}\right)= \begin{cases}F(0,0)-\gamma & k_{x}=k_{y}=0 \\
F\left(k_{x}, k_{y}\right) & \text { elsewhere }\end{cases} \\
& F_{\delta}\left(k_{x}, k_{y}\right)= \begin{cases}F(0,0)-\delta & k_{x}=k_{y}=0 \\
F\left(k_{x}, k_{y}\right) & \text { elsewhere }\end{cases}
\end{aligned}
$$

are irreducible over the set of complex numbers.

The following theorem is on the theory of POCS:

Theorem 10 (Youla and Webb [35]): Let $H$ be a Hilbert space with elements $f, g, \cdots$, etc., a zero vector $\phi$, and an inner product $(x, y)$. Furthermore, let $C_{0}$, the intersection of closed convex subsets $C_{1}, \cdots, C_{m}$ of $H$ given by

$$
C_{0}=\bigcap_{i=1}^{m} C_{i}
$$

be non-empty. Consider the composition operator

$$
T=T_{m} T_{m-1} \cdots T_{1}
$$

where

$$
T_{i}=1+\lambda_{i}\left(P_{i}-1\right)
$$

and $P_{i}$ is a projection operator onto $C_{i}$. Then, for every $x \in$ $H$ and every choice of relaxation constants $\lambda_{1}, \cdots, \lambda_{m}$ in the interval

$$
0<\lambda_{i}<2, \quad 1 \leq i \leq m
$$

the sequence $\left\{T^{n} x\right\}$ converges weakly to a point in $C_{0}$. If $H$ is finite dimensional, the convergence is strong. In addition, if one of the $C_{i}$ 's, e.g., $C_{m}$, is finite dimensional by setting

$$
\lambda_{m}=1
$$

we are guaranteed of strong convergence, even though $H$ might be infinite dimensional.

APPENDIX B

Proof and Corollaries of Theorem 8

This appendix includes proof of Theorem 8 and a recursive proof for a special case of it. We begin with the proof of Theorem 8

Proof: To show that there is a unique polynomial which satisfies (28), we have to show that there are no polynomials in $\Pi_{\left(N_{w}, N_{2}\right)}$ which vanish at all the interpolation points $\cup A_{i}$. Suppose, on the contrary, that there is a polynomial $q(w$,

${ }^{17}$ Definition of topological dimension is given in Appendix $\mathrm{C}$. $z) \in \Pi_{\left(N_{w}, N_{z}\right)}$ which vanishes at all the interpolation points. Since $q$ has $M_{w}^{(0)} N_{z}+N_{w} M_{z}^{(0)}+1$ common zeros with $C_{0}$, by the modified version of Bezout's theorem, $C_{0}$ must be a factor of $q(w, z)$. That is,

$$
q(w, z)=C_{0}(w, z) q^{(1)}(w, z)
$$

where $q^{(1)}(w, z)$ is a polynomial of maximum degree $N_{w}-$ $M_{w}^{(0)}$ in $w$ and $N_{z}-M_{z}^{(0)}$ in $z$. Furthermore, since by hypothesis, none of the interpolation points on $C_{1}$ are on $C_{0}$ and $q(w, z)$ has $1+M_{z}^{(1)}\left(N_{w}-M_{w}^{(0)}\right)+M_{w}^{(1)}\left(N_{z}-M_{z}^{(0)}\right)$ common zeros with $C_{1}, q^{(1)}(w, z)$ must also have the same number of common zeros with $C_{1}$. Taking into account the irreducibility of $C_{1}$, by modified version of Bezout's theorem, $C_{1}$ must be a factor of $q^{(1)}(w, z)$, and hence $q(w, z)$.

Repeating the preceding argument for $C_{2}, \cdots, C_{N_{C}-1}$, we get

$$
q(w, z)=C_{0}(w, z) \cdots C_{N_{c}-1}(w, z) q^{\left(N_{c}\right)}(w, z)
$$

where $q^{(q)}(w, z)$ has the maximum degree $N_{w}-\Sigma_{i=0}^{N_{c}-1} M_{w}^{(i)}$ in $w$ and maximum degree $N_{z}-\Sigma_{i=0}^{N_{c}-1} M_{z}^{(i)}$ in $z$ and has 1 $+M_{w}^{\left(N_{c}\right)}\left(N_{z}-\Sigma_{k=0}^{N_{c}-1} M_{z}^{(k)}\right)+M_{z}^{\left(N_{c}\right)}\left(N_{w}-\Sigma_{k=0}^{N_{c}-1} M_{w}^{(k)}\right)$ common zeros with $C_{N_{c}}$. Since $C_{N_{c}}$ is irreducible, by modified version of Bezout's theorem it must be a factor of $q^{\left(N_{c}\right)}(w, z)$. This contradicts the hypothesis, since by inequalities (28) and (6) the degree of $C_{N_{c}}$ in either $w$ or $z$ is larger than that of $q^{\left(N_{c}\right)}(W, z)$.

There is an alternate proof for a special case of Theorem 8 where all the sampling curves are chosen to be lines passing through origin. This proof is not necessarily simpler than the one we have given for the most general case earlier. However, it provides a recursive way of computing the coefficients of the polynomial under consideration. This special case of Theorem 8 can be stated in the following manner.

Corollary 1 : Let $I_{0}, \cdots, I_{N}$ be distinct lines with $I_{i}$, the $i$ th line, defined by

$$
z=\alpha_{i} W \quad \alpha_{i} \neq 0
$$

and consider arbitrary distinct points on $I_{i}$ given by

$$
\left\{\left(w_{i}^{(i)}, z_{j}^{(i)}\right) \mid j=0, \cdots, 2 i\right\}
$$

where the ordering of the lines is arbitrary. If none of the interpolation points is equal to $(0,0)$, the common intersection of all lines, then for any data set

$$
\left\{t_{i}^{(i)} \mid 0 \leq j \leq 2 i ; 0 \leq i \leq N\right\}
$$

there is no more than one bivariate polynomial of the form

$$
p(w, z)=\sum_{i=0}^{N} \sum_{j=0}^{N} a(i, j) w^{i} z^{j}
$$

such that

$$
p\left(w_{j}^{(i)}, z_{i}^{(i)}\right)=t_{j}^{(i)} \quad 0 \leq j \leq 2 i ; \quad 0 \leq i \leq N .
$$

The recursive proof of the preceding corollary is as follows.

Proof: Substituting the equation of the $k$ th line, $l_{k}$ into $p(w, z)$, we get

$$
\begin{aligned}
p\left(w, \alpha_{k} w\right) & =\sum_{i=0}^{N} \sum_{i=0}^{N} a(i, j) w^{i}\left(\alpha_{k} w\right)^{j} \\
& =\sum_{i=0}^{2 N} b_{i}^{(k)} w^{i}
\end{aligned}
$$


where

$$
b_{i}^{(k)}= \begin{cases}\sum_{m=0}^{i} a(i-m, m) \alpha_{k}^{m} & 0 \leq i \leq N \\ \sum_{m=i-N}^{N} a(i-m, m) \alpha_{k}^{m} & N \leq i \leq 2 N .\end{cases}
$$

For an arbitrary integer $s \geq-1$, we can split the summation in (34) in the following manner:

$$
\sum_{i=s+1}^{2 N-s-1} b_{i}^{(k)} w^{i}=p\left(w, \alpha_{k} w\right)-\sum_{i=0}^{s} b_{i}^{(k)} w^{i}-\sum_{i=2 N-s}^{2 N} b_{i}^{(k)} w^{i} .
$$

Setting $s=-1$, using the $2 N+1$ points of $I_{N}$, we can uniquely determine $b_{i}^{(N)}$ for $0 \leq i \leq 2 N$. In particular, considering (35), the values of $b_{0}^{(N)}$ and $b_{2 N}^{(N)}$ enable us to determine $a(0$, $0), a(N, N)$, and $b_{2 N}^{(j)}$ for $0 \leq j \leq N-1$. This is because, from (35), we have

$$
\begin{aligned}
& b_{0}^{(k)}=a(0,0) \\
& b_{2 N}^{(k)}=a(N, N) \alpha_{k}^{N} .
\end{aligned}
$$

Similarly, by setting $s=0$ in (36) and using the $2 N-1$ points on $I_{N-1}$, we can uniquely specify $b_{i}^{(N-1)}$ for $1 \leq i \leq 2 N-$ 1. This can be done because the determinant

$$
\left|\begin{array}{cccc}
x_{0} & x_{0}^{2} & \cdots & x_{0}^{2 N-1} \\
x_{1} & x_{1}^{2} & \cdots & x_{1}^{2 N-1} \\
& \vdots & \cdots & \vdots \\
x_{2 N-2} & x_{2 N-2}^{V_{2}} & \cdots & x_{2 N-2}^{2 N-1}
\end{array}\right|
$$

is nonzero as long as the $x_{i}^{\prime}$ s are different from each other and from zero. Now we can utilize the values of $b_{1}^{(N)}$ and $b_{1}^{(N-1)}$ together with (35) to find $a(0,1)$ and $a(1,0)$. More specifically, from (35) we have:

$$
b_{1}^{(k)}=\alpha_{k} a(0,1)+a(1,0) .
$$

Letting $k=N, N-1$ in the preceding equation, we can uniquely specify $a(0,1), a(1,0), a(1,0)$, and hence $v_{1}^{k}$ for $0 \leq$ $k \leq N-2$.

In a similar manner, the values of $b_{2 N-1}^{(N)}$ and $b_{2 N-1}^{(N-1)}$ can be used to find $a(N, N-1)$ and $a(N-1, N)$, and hence $b_{2 N-1}^{(k)}$. More specifically, from (35) we get

$$
b_{2 N-1}^{(k)}=\alpha_{k}^{N} a(N-1, N)+\alpha_{k}^{N-1} a(N, N-1) .
$$

The determinant of the preceding system of equations for $k=N, N-1$ is given by

$$
\left|\begin{array}{ll}
\alpha_{N-1}^{N} & \alpha_{N-1}^{N-1} \\
\alpha_{N}^{N} & \alpha_{N}^{N-1}
\end{array}\right|=-\alpha_{N-1}^{N-1} \alpha_{N}^{N-1}\left|\begin{array}{ll}
\alpha_{N-1} & 1 \\
\alpha_{N} & 1
\end{array}\right| .
$$

Taking into account that $\alpha_{k} \neq 0$, the preceding determinant is guaranteed to be nonzero. Thus the coefficients a $(N, N$ - 1) and $a(N-1, N)$ can be specified uniquely.

Repeating the preceding procedure for $s=1, \cdots, 2 \mathrm{~N}$ -1 , we can find all the coefficients $a(i, j)$. More specifically, at the $s$ th stage, we know $b_{2 N-s}^{(k)}$ and $b_{s}^{(k)}$ for $0 \leq k \leq N$, and using the $2(N-s)-1$ points of the line $I_{N-s-1}$, we can find $b_{i}^{(N-s-1)}$ for $s+1 \leq i \leq 2 N-s-1$. These values will enable us to uniquely specify the coefficients

$$
\{a(i, j) \mid i+j=s+1,2 N-s-1\}
$$

and hence $b_{2 N-s-1}^{(k)}$ and $b_{s+1}^{(k)}$ for $0 \leq k \leq N-s-2$. Consequently, we can completely and uniquely determine all the coefficients of $p(w, z)$.

Thus the preceding proof not only shows that the appropriate set of interpolation points on lines passing through the origin results in a unique solution, but also provides us with a recursive method to find the coefficients of the polynomial under consideration. The recursive algorithm described by the preceding proof essentially consists of $s$ $=-1, \cdots, 2 N+1$ stages, and at each stage two Van der Monde linear system of equations are solved. More specifically, at the sth stage, the algorithm first uses the computed values of $b_{2 N-s}^{(k)}$ and $b_{s}^{(k)}$ for $0 \leq k \leq N$, together with the $2(N-s)-1$ samples on the line $I_{N-s-1}$, in order to find $b_{i}^{(N-s-1)}$ for $s+1 \leq i \leq 2 N-s-1$ by solving a $(2 N-s)$ $\times(2 N-s)$ linear system of equations. It then uses these values in (35) to find the coefficients $\{a(i, j) \mid i+j=s+1$, $2 N-s-1\}$ by solving a $(k+2) \times(k+2)$ system of linear equations.

Since lines passing through origin in the $w-z$ domain correspond to lines of unit slope in the $w-z$ domain, the recursive algorithm outlined by the proof of Corollary 1 can be used to reconstruct BL.P signals from their samples on line of unit slope.

Another special case of Theorem 8 resulting in a recursive algorithm occurs when all the sampling curves are chosen to be of the form $z=\alpha w^{m}$, with $m$ being a fixed positive integer. Since curves of the form $z=\alpha w^{m}, m>0$ in the $w-z$ domain correspond to lines of positive integer slope in the $w-z$ domain, the recursive proof of this case can be used, at least in principle, to reconstruct multidimensional BLP signals from their samples on parallel lines of positive integer slope. The proof is considerably more complex than that of Corollary 1 and is included in [30].

Another corollary of Theorem 8 can be derived by taking into account that curves of the form given by (8) are irreducible and that they correspond to lines of rational slope in the $x-y$ domain. As indicated in section III-A.2, this corollary is the basis for the semi-implicit sampling scheme.

Corollary 2: Consider a bandlimited continuous-time periodic signal $f(x, y)$ with period one in the $x$ and $y$ directions and Fourier series representation:

$$
f(x, y)=\sum_{k_{x}=-N_{x}}^{N_{x}} \sum_{k_{y}=-N_{y}}^{N_{y}} F\left(k_{x}, k_{y}\right) e^{i 2 \pi\left(k_{x} x+k_{y} y\right)} .
$$

Let $I_{0}, \cdots, I_{N}$ be distinct lines in the $x-y$ plane with $I_{i}$, the $i$ th line given by

$M_{y}^{(i)} y=M_{x}^{(i)} x+\beta_{i} \quad$ or $\quad M_{y}^{(i)} y+M_{x}^{(i)} x=\beta_{i} \quad M_{x}^{(i)}, M_{y}^{(i)}>0$

where $M_{x}^{(i)}$ and $M_{y}^{(i)}$ are relatively prime positive integers. Let $N_{l}$ be an integer satisfying either one of the following:

$$
\begin{aligned}
& 2 N_{x}<\sum_{i=0}^{N_{l}} M_{x}^{(i)} \\
& 2 N_{y}<\sum_{i=0}^{N_{l}} M_{y}^{(i)} .
\end{aligned}
$$

Suppose that the set of

$$
S(i)=M_{y}^{(i)}\left(2 N_{x}-\sum_{k=0}^{i-1} M_{x}^{(k)}\right)+M_{x}^{(i)}\left(2 N_{y}-\sum_{k=0}^{i-1} M_{y}^{(k)}\right)+1
$$


arbitrary distinct samples on $l_{i}$, is given by

$$
\left\{\left(x_{j}^{(i)}, y_{j}^{(i)}\right) \mid 0 \leq i \leq N_{l}, 0 \leq j<S(i)\right\} .
$$

If none of the interpolation points given by (47) are on the intersection of two or more of the $c_{i}^{\prime}$ 's, then for any data set

$$
\left\{t_{i}^{(i)} \mid 0 \leq j<S(i)\right\}
$$

there exists no more than one signal of the form given by (43) which satisfies

$$
f\left(x_{j}^{(i)}, y_{j}^{(i)}\right)=t_{j}^{(i)} \quad 0 \leq i \leq N_{1}, 0 \leq j<S(i) .
$$

APPENDIX C

A Result in Algebraic Geometry

In this Appendix, we will briefly go over few definitions, and then prove a result in algebraic geometry, which is used in the proof of Theorem 9.

Consider the polynomials

$$
p_{i}\left(x_{0}, \cdots, x_{n}\right)=0, \quad i=1, \cdots, r
$$

defining an irreducible algebraic set $V$ over $\mathcal{K}$. Let us denote the $r \times n$ Jacobian matrix associated with $V$ by $\left(\partial f_{i} / \partial x_{j}\right)$. Points $x^{\prime}$ of $V$ at which the Jacobian matrix assumes is maximum rank are called ordinary points. Any point of $V$ which is not ordinary is said to be singular [42]. If the singular points of $V$ are removed, we obtain a manifold whose dimension defines the topological dimension of $V .{ }^{18}$ The dimension of a reducible algebraic set is defined to be the maximum of topological dimensions of its various irreducible components.

The complex topological dimensions of $V \subset C^{n}$, which is defined to be the dimension of its associated complex manifold, is also given by [44]:

$$
n-\max _{P}\left(\frac{\partial f_{i}}{\partial x_{j}}\right)_{P}
$$

where $P$ ranges over the points in $V$. By definition, the real dimension of $V$ is twice its complex dimension. For convenience, we will use the term topological dimension to denote real dimension unless specified otherwise. If $V(R)$ denotes the real part of $V \subset C^{n}$, we have [45]

$$
\operatorname{dim}_{\text {top }} V(R) \leq \frac{1}{2} \operatorname{dim}_{\text {top }} V
$$

where $\operatorname{dim}_{\text {top }}$ denotes the topological dimension. $V(R)$ is said to be of maximal topological dimension if its dimension is exactly half the dimension of $V$.

We are now ready to prove the following theorem, which is ultimately used in the proof of Theorem 9.

Theorem $11^{19}$ : Let $V \subset C^{N}$ be the set of complex zeros of polynomials $f_{1}, \cdots, f_{r}$. Then if $V$ is irreducible and $V(R)$, the real points of $V$, have maximal topological dimension, then $V(R)$ is Zariski dense in $V$. That is, every polynomial that vanishes on $V(R)$ must vanish on all of $V$.

Proof: We will prove the preceding result by contradiction. ${ }^{19}$ If a polynomial $f$ vanishes on $V(R)$ and does not vanish on $V$, then let $W \subset V$ be the set of real and complex zeros of $f$ which are in $V$. Since $V$ is irreducible, and $W$ is

\footnotetext{
${ }^{18}$ Precise definition of manifolds is included in [43].

${ }^{19}$ This theorem and its proof were suggested by Prof. M. Artin at MIT.
}

a proper subset of $V$,

$$
\operatorname{dim}_{\text {top }} W<\operatorname{dim}_{\text {top }} V
$$

Since the real part of $W$ and $V$ are the same, then

$$
\operatorname{dim}_{\text {top }} W(R)=\operatorname{dim}_{\text {top }} V(R) \text {. }
$$

Using the preceding equation and the assumption of maximal topological dimension for $V(R)$, we get

$$
\operatorname{dim}_{\text {top }} W(R)=\frac{1}{2} \operatorname{dim}_{\text {top }} V \text {. }
$$

Furthermore, as we mentioned earlier,

$$
\operatorname{dim}_{\text {top }} W(R) \leq \frac{1}{2} \operatorname{dim}_{\text {top }} W .
$$

From equations (50) and (51) we conclude that

$$
\operatorname{dim}_{\text {top }} V \leq \operatorname{dim}_{\text {top }} W
$$

which contradicts inequality (49).

APPENDIX D

Proof of Theorem 9

This appendix includes the proof of Theorem 9.

Proof: The outline of the proof is as follows. We will first use the first condition to show that $\tilde{B}(R)$ is an irreducible algebraic set over reals. Then either zeros of $\bar{p}$ are of measure zero in $\tilde{B}(R)$ or all points in $\tilde{B}(R)$ are zeros of $\tilde{p}$. Since our objective is to prove that real zeros of $\tilde{p}$ are of measure zero in $\tilde{B}(R)$, all we have to show is that there is at least one point in $\bar{B}(R)$ at which $\bar{p}$ does not vanish. As we will see, the second condition of the theorem will be used to show this.

To begin, notice that the polynomials $g_{\gamma}\left(W_{1}, W_{2}\right)$ and $g_{\delta}\left(W_{1}, W_{2}\right)$ are related to our BLP signal $f(x, y)$ via the change of variables

$$
\begin{aligned}
& W_{1}=e^{j 2 \pi x} \\
& W_{2}=e^{j 2 \pi y}
\end{aligned}
$$

in the following manner:

$$
\begin{aligned}
& f(x, y)-\gamma=W_{1}^{-N} W_{2}^{-N} g_{\gamma}\left(W_{1}, W_{2}\right) \\
& f(x, y)-\delta=W_{1}^{-N} W_{2}^{-N} g_{\delta}\left(W_{1}, W_{2}\right) .
\end{aligned}
$$

Thus there is a one to one correspondence between zeros of $g_{\gamma}\left(W_{1}, W_{2}\right)$ (resp. $g_{\delta}\left(W_{1}, W_{2}\right)$ ) and $\tilde{A}_{\gamma}(C)$ (resp. $\bar{A}_{\delta}(C)$. Therefore the second condition of the theorem implies that $\bar{A}_{\gamma}$ and $\tilde{A}_{\delta}$ are also irreducible. Considering (19), since $\bar{B}(C)$ is the Cartesian product of $\bar{A}_{\gamma}(C)$ 's and $\tilde{A} \delta(C)$ 's, we can conclude that $\tilde{B}(C)$ is also irreducible over complex numbers.

Similarly, since $\bar{A}_{\gamma}(R)$ and $\bar{A}_{\delta}(R)$ have maximal topological dimensions, so does $\tilde{B}(R)$. Therefore, considering Theorem 11 of Appendix $(C)$ and taking into account that $\tilde{B}(C)$ is irreducible, we can conclude that $\tilde{B}(R)$ is Zariski dense in $\tilde{B}(C)$. This means that every polynomial that vanishes on $\tilde{B}(R)$ vanishes on all of $\bar{B}(C)$. This, together with the fact that $\bar{B}(C)$ is irreducible, implies that $\bar{B}(R)$ is irreducible over complex numbers and thus the reals. Therefore, in order to show that the real zeros of $\bar{p}\left(w_{1}, \cdots, v_{(2 N+1)^{2}}\right)$ are of measure zero in $\tilde{B}(R)$, we merely have to show that there exists at least one point in $\tilde{B}(R)$ at which $\bar{p}$ does not vanish. That is, $\tilde{p}$ does not vanish identically on $\tilde{B}(R)$.

Since, by hypothesis, $g_{\gamma}\left(W_{1}, W_{2}\right)$ and $g_{\delta}\left(W_{1}, W_{2}\right)$ are irreducible over complex numbers, taking into account mod- 
ified version of Bezout's theorem, we can conclude that any $8 N^{2}+1$ samples of $A_{\gamma}$ will enable us to specify $f(x, y)-\gamma$ to within a scale factor [46]. This means the $\left(8 N^{2}+1\right) \times(2 N$ $+1)^{2}$ matrix associated with any $8 N^{2}+1$ points of $A_{\gamma}, D$ has rank $(2 N+1)^{2}-1$, and its null vector is specified by the coefficients of $f(x, y)-\gamma$. Similarly, $D_{\delta}$, the $8 N^{2}+1 \times$ $(2 N+1)^{2}$ matrix associated with any $8 N^{2}+1$ points of $A_{\delta}$, has rank $(2 N+1)^{2}-1$, and its null vector is given by the coefficients of $f(x, y)-\delta$. Since by hypothesis $\gamma \neq \delta$, the direction of the null vectors of $D_{\gamma}$ and $D_{\delta}$ are different from each other. Therefore there exists at least one combination of $k$ rows from $D_{\gamma}$ and $(2 N+1)^{2}-k$ rows from $D_{\delta}$, which result in a full rank $(2 N+1)^{2} \times(2 N+1)^{2}$ matrix with nonzero determinant $\tilde{p}$. Hence $\tilde{p}$ does not vanish identically on $\tilde{B}(R)$.

Since $\tilde{B}(R)$ is irreducible over reals and $\tilde{p}$ does not vanish on it identically, the real zeros of $\tilde{p}$ must be of measure zero in $\tilde{B}(R)$. QED.

\section{REFERENCES}

[1] S. R. Curtis and A. V. Oppenheim, "Reconstruction of multidimensional signals from zero crossings," J. Optical Soc. Amer., vol. 4, pp. 221-231, Jan. 1987.

[2] F. E. Bond and C. R. Cahn, "On sampling the zeros of bandwidth limited signals," IRE Trans. Informat. Theory, vol. IT-4, pp. 110-113, 1958.

[3] A. Sekey, "A computer simulation study of real-zero interpolation," IEEE Trans. Audio Electroacoustics, vol. 18, no. 1, pp. 43-54, Mar. 1970.

[4] H. B. Voelcker, "Toward a unified theory of modulatio-Part ii: Zero manipulation," Proc. IEEE, vol. 54, no. 5, pp. 735-755, May 1966.

[5] B. F. Logan, Jr., "Information in zero crossing of bandpass signals," Bell Syst. Tech. J., vol. 56, pp. 487-510, Apr. 1977.

[6] D. Marr, S. UlIman, and T. Poggio, "Bandpass channels, zero crossing and early visual information processing," $J$. Optical Soc. Amer., vol. 69, pp. 914-916, June 1979.

[7] I. Bar-David, "An implicit sampling theorem for bounded bandlimited functions," Inform. Control, vol. 24, pp. 36-44, 1974.

[8] J. S. Lim, A. V. Oppenheim, and S. R. Curtis, "Signal synthesis and reconstruction from partial fourier domain information," J. Optical Soc. Amer., vol. 73, no. 11, pp. 1413-1420, Nov. 1983.

[9] J. S. Lim, M. H. Hayes, and A. V. Oppenheim, "Signal reconstruction from phase or magnitude," IEEE Trans. Acoust. Speech Signal Processing, vol. 28, no. 6, pp. 672-680, Dec. 1980

[10] M. H. Hayes, "The reconstruction of a multidimensional sequence from the phase or magnitude of its fourier transform," IEEE Trans. Acoust. Speech Signal Processing, vol. 30, no. 6, pp. 145-154, Dec. 1982.

[11] J. S. Lim, P. L. Van Hove, M. Hayes, and A. V. Oppenheim, "Signal reconstruction from signed fourier transform magnitude," IEEE Trans. Acoust. Speech Signal Processing, vol. 31 , no. 5 , pp. $1286-1293$, Oct. 1983

[12] J. L. C. Sanz and T. S. Haung, "Unique reconstruction of a bandlimited multidimensional signal from phase or magnitude," J. Optical Soc. Amer., vol. 73, no. 11, pp. 1446-1450, Nov. 1983.

[13] M. H. Hayes and ). H. McClellan, "Reducible polynomials in more than one variable," Proc. IEEE, vol. 70 , no. 2 , pp. 197198, Feb. 1982.

[14] D. Marr and E. Hildreth, "Theory of edge detection," Proc. R. Soc. Lond. B, vol. 207, pp. 187-217, 1980.

[15] J. E. Bondaryk, "Reconstruction of nonlinearly distorted images from zero crossings," M.S. thesis, Massachusetts Institute of Technology, 1987.

[16] D. Rotem and Y. Y. Zeevi, "Image reconstruction from zero crossings," IEEE Trans. Acoust. Speech Signal Processing, vol. 34, no. 5, pp. 1269-1277, Oct. 1986.

[17] A. V. Oppenheim, S. R. Curtis, and J. S. Lim, "Signal reconstruction from Fourier transform sign information," IEEE
Trans. Acoust. Speech Signal Processing, vol. 33, no. 3, pp. 643-657, June 1985

[18] A. Zakhor and D. Izraelevitz, "A note on the sampling of zerocrossings of two-dimensional signals," Proc. IEEE, vol. 74, no. 9, pp. 1285-1287, Sept. 1986.

[19] C. A. Michelli, "Algebraic aspects of interpolation," in Approximation Theory Short Course. Providence, Rl: Amer. Math. Soc., 1986.

[20] S. Karlin, Total Positivity. Stanford, CA: Stanford University Press, 1968.

[21] S. Karlin and W. J. Sudden, Tchebycheff Systems: With Applications in Analysis and Statistics. New York: Interscience, 1966.

[22] M. G. Krein, "The ideas of P. L. Chebyshev and A. A. Markoff in theory of limiting values of integrals and their further developments, AMS Transl. Ser., vol. 2, no. 12, pp. 1-122, 1951.

[23] G. G. Lorentz and R. A. Lorentz, "Multivariate interpolation," in Rational Approximation and Interpolation, E. B. Saff, P. R. Graves-Morris, and R. S. Varga, Eds., Tampa, FL: Springer-Verlag, pages 136-144, Dec. 1983; Proc. United Kingdom-United States Conf.

[24] K. C. Chung and T. H. Yao, "On lattices admitting unique Lagarange interpolations," SIAM J. Numerical Anal., vol. 14, no. 4, pp. 735-743, Sept. 1977.

[25] M. Gasca and E. Lebron, "A note on recurrence interpolation formulae for certain sets of points in $r^{k}$, , ISNM, Numerical Methods of Approximation Theory, vol. 67, no. 7, pp. 77-85, 1984.

[26] R. A. Nicolaides, "On a class of finite elements generated by Lagrange interpolation," SIAM I. Numerical Anal., vol. 9, no. 3, pp. 435-445, Sept. 1972.

[27] M. Gasca and I. I. Maeztu, "On Lagrange and hermite interpolation in $r^{k}, "$ Numerische Mathematik, vol. 39, pp. 1-14, 1982.

[28] E. W. Cheney, Multivariate Approximation Theory: Selected Topics. CBMS-NSF Regional Conference Series in Applied Mathematics, 1986.

[29] F. Stenger, "Kronecker product extensions of linear operations," SIAM J. Numerical Anal., vol. 5, no. 2, pp. 422-435, June 1968.

[30] A. Zakhor, "Reconstruction of multidimensional signals from multiple level threshold crossings, Ph.D. thesis, Massachusetts Institute of Technology, 1987.

[31] E. Kaltofen, "Factorization of polynomials," Computing Suppl., vol. 4, pp. 95-113, 1982.

[32] William Fulton, Algebraic Curves. New York, NY: W. A. Benjamin, 1969.

[33] Monson $\mathrm{H}$. Hayes, "Signal reconstruction from phase or magnitude," Ph.D. thesis, Massachusetts Institute of Technology, 1981.

[34] A. Vistoli, private communication.

[35] D. C. Youla and $\mathrm{H}$. Webb, "Image restoration by the method of convex projections: Part 1-Theory," IEEE Trans. Medical Imag., vol. 1, no. 2, pp. 81-94, Oct. 1982.

[36] T. S. Motzkin and I. Shoenberg, "The relaxation method for linear inequalities," Can. J. Math., vol. 6, no. 3, pp. 393-404, 1954.

[37] Russell M. Mersereau, "Digital reconstruction of multidimensional signals from their projections," Ph.D. thesis, Massachusetts Institute of Technology, 1974.

[38] T. S. Haung, "Coding of two-tone images," IEEE Trans. Commun., vol. 25, no. 11, pp. 1406-1424, Nov. 1977.

[39] D. N. Graham, "Image transmission by two-dimensional contour coding," IEEE Trans. Commun., vol. 55, no. 3, pp. 336346.

[40] R. J. Walker, Algebraic Curves. New York: Springer-Verlag, 1978.

[41] A. Mostowski and M. Stark, Introduction to Higher Algebra. New York: MacMillan, 1964.

[42] Solomon Lefschetz, Algebraic Geometry. Princeton University Press, 1953.

[43] Victor Guillemin and Alan Pollack, Differential Topology. New York: Prentice-Hall, 1974.

[44] W. V. D. Hodge and D. Pedoe, Methods of Algebraic Geometry. Cambridge, UK: Cambridge University Press, 1968.

[45] Tom M. Apostol, Mathematical Analysis. Reading, MA: Addison-Wesley, 1974. 
[46] S. R. Curtis, "Reconstruction of multidimensional signals from zero crossings," Ph.D. thesis, Massachusetts Institute of Technology, 1985.

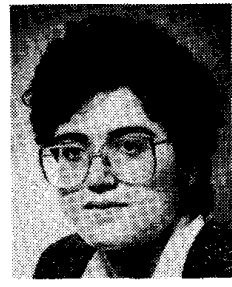

Avideh Zakhor (Member, IEEE) received the B.S. degree from California Institute of Technology, Pasadena, and the S.M. and Ph.D. degrees from Massachusetts Institute of Technology, Cambridge, all in electrical engineering, in 1983, 1985, and 1987, respectively. Her doctoral thesis was on sampling and reconstruction schemes for multidimensional signals.

In 1988 she joined the Faculty at the University of California at Berkeley, where she is currently Assistant Professor in the Department of Electrical Engineering and Computer Sciences. Her research interests are in the general area of signal processing and its applications to images, to video, and to biomedical data. She has been a consultant to a number of industrial organizations in the areas of signal processing, communications and medical imaging, and has two pending patents on NMR signal processing.
Dr. Zakhor has been a Hertz Fellow, a General Motors scholar, and an Atlantic College scholar. She received the Henry Ford Engineering Award and the Caltech Prize in 1983. She is a member of Tau Beta Pi, Sigma Xi, and the ASSP Society of the IEEE.

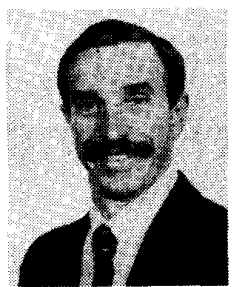

Alan V. Oppenheim (Fellow IEEE) received the S.B. and S.M. degrees in 1961, and the Sc.D. degree in 1964, all in electrical engineering, from the Massachusetts Institute of Technology, Cambridge.

In 1964 he joined the faculty at MIT, where he is currently Professor of Electrical Engineering and Computer Science. His research interests are in the general area of signal processing and its application to speech, image, and seismic data processing. He is coauthor and editor of several texts on signal processing. Dr. Oppenheim has received a number of awards for outstanding research and teaching, including the 1988 IEEE Education Medal. $\mathrm{He}$ is a member of the National Academy of Engineering, Tau Beta $\mathrm{Pi}$, Eta Kappa Nu, and Sigma Xi. 